\title{
SHARP ENERGY ESTIMATES FOR NONLINEAR FRACTIONAL DIFFUSION EQUATIONS
}

\author{
XAVIER CABRÉ AND ELEONORA CINTI
}

\begin{abstract}
We study the nonlinear fractional equation $(-\Delta)^{s} u=f(u)$ in $\mathbb{R}^{n}$, for all fractions $0<s<1$ and all nonlinearities $f$. For every fractional power $s \in(0,1)$, we obtain sharp energy estimates for bounded global minimizers and for bounded monotone solutions. They are sharp since they are optimal for solutions depending only on one Euclidian variable.

As a consequence, we deduce the one-dimensional symmetry of bounded global minimizers and of bounded monotone solutions in dimension $n=3$ whenever $1 / 2 \leq$ $s<1$. This result is the analogue of a conjecture of De Giorgi on one-dimensional symmetry for the classical equation $-\Delta u=f(u)$ in $\mathbb{R}^{n}$. It remains open for $n=3$ and $s<1 / 2$, and also for $n \geq 4$ and all $s$.
\end{abstract}

\section{INTRODUCTION AND RESULTS}

In this paper we establish energy estimates for some bounded solutions of the fractional nonlinear equation

$$
(-\Delta)^{s} u=f(u) \text { in } \mathbb{R}^{n},
$$

for every $0<s<1$, where $f: \mathbb{R} \rightarrow \mathbb{R}$ is a $C^{1, \gamma}$ function for some $\gamma>\max (0,1-2 s)$.

In [4], we considered the case $s=1 / 2$ and established sharp energy estimates for bounded global minimizers in every dimension $n$, and for bounded monotone solutions in dimension $n=3$. As a consequence, we deduced one-dimensional (or 1-D) symmetry for these types of solutions in dimension $n=3$.

This result about 1-D symmetry is the analogue of a conjecture of De Giorgi for the Allen-Cahn equation $-\Delta u=u-u^{3}$ in $\mathbb{R}^{n}$. More precisely, in 1978 De Giorgi conjectured that the level sets of every bounded solution of the Allen-Cahn equation which is monotone in one direction must be hyperplanes, at least if $n \leq 8$. That is, such solutions depend only on one Euclidean variable. The conjecture was proven to be true in dimension $n=2$ by Ghoussoub and Gui [16] and in dimension $n=3$ by

Both authors were supported by grants MTM2008-06349-C03-01 (Spain), MTM2011-27739-C0401 (Spain), and 2009SGR345 (Catalunya). The second author was partially supported by University of Bologna (Italy), funds for selected research topics. 
Ambrosio and the first author [3]. For $4 \leq n \leq 8$, if $\partial_{x_{n}} u>0$, and assuming the additional condition

$$
\lim _{x_{n} \rightarrow \pm \infty} u\left(x^{\prime}, x_{n}\right)= \pm 1 \quad \text { for all } x^{\prime} \in \mathbb{R}^{n-1},
$$

it has been established by Savin [23. More recently, a counterexample to the conjecture for $n \geq 9$ has been found by Del Pino, Kowalczyk, and Wei [13].

In this paper (see Theorem 1.5 below), we establish the one-dimensional symmetry of bounded global minimizers and of bounded monotone solutions of (1.1) in dimension $n=3$ for every $1 / 2 \leq s<1$. This is the analogue of the conjecture of De Giorgi for the operator $(-\Delta)^{s}$. Our result applies to all nonlinearities $f \in C^{1, \gamma}$, $\gamma>\max \{0,1-2 s\}$.

In dimension $n=3$, in 4 4 we proved the same result for $s=1 / 2$. For $n=3$ and $0<s<1 / 2$ the question remains open, as well as for $n \geq 4$ and any $0<s<1$. For $n=2$ and $s=1 / 2$, the one-dimensional symmetry of bounded stable solutions of (1.1) was proven by the first author and Solà-Morales [7]. The same result in dimension $n=2$ for every fractional power $0<s<1$ has been established by the first author and Sire [6] and by Sire and Valdinoci [28]. Recall that the class of stable solutions includes all global minimizers, as well as all monotone solutions.

The existence of 1-D monotone solutions (also called "layers") has been established by the first author and Sire in [5, 6] for all nonlinearities $f$ (not necessarily odd) for which its primitive (up to a sign) is a potential of "double-well type". In fact, [5, 6] establish that this is a necessary and sufficient condition on $f$ for a 1-D monotone solution to exist. Our 1-D symmetry result for monotone solutions in $\mathbb{R}^{3}$ will use both the 1-D symmetry result in $\mathbb{R}^{2}$ of [6] and (since it applies to all nonlinearities $f$ ) the necessary conditions on $f$ proved in [5] for a monotone solution in $\mathbb{R}$ to exist.

As in [4], a crucial ingredient in the proof of 1-D symmetry is a sharp energy estimate for bounded global minimizers and for bounded monotone solutions. These are Theorems 1.2 and 1.4. Our estimates are sharp since they are optimal for solutions depending only on one Euclidian variable.

The classical connection between the Allen-Cahn equation and minimal surfaces (or the perimeter functional) is the motivation behind the conjecture of De Giorgi. The following are some results in this direction but concerning the fractional Laplacian. In [17] M.d.M. González proved that an energy functional related to fractional powers $s$ of the Laplacian (for $1 / 2<s<1$ ) $\Gamma$-converges to the classical perimeter functional. The same result for $s=1 / 2$ had been proven by Alberti, Bouchitté, and Seppecher in [2]. In [10] Caffarelli and Souganidis prove that scaled threshold dynamics-type algorithms corresponding to fractional Laplacians converge to certain moving fronts. 
More precisely, when $1 / 2 \leq s<1$ the resulting interface moves by a weighted mean curvature, while for $0<s<1 / 2$ the normal velocity is nonlocal of fractional-type. Later, Caffarelli, Roquejoffre, and Savin [8] set up the theory of nonlocal $s$-minimal surfaces for $0<s<1 / 2$. Palatucci, Savin, and Valdinoci [22, 24, 25] have established precise relations between $s$-minimal surfaces and the interfaces of semilinear phase transition equations driven by the fractional Laplacian. See Remark 1.6 for more comments on s-minimal surfaces.

To study the nonlocal problem (1.1), we will realize it as a local problem in $\mathbb{R}_{+}^{n+1}$ with a nonlinear Neumann condition. More precisely, Caffarelli and Silvestre [9] proved that $u$ is a solution of problem (1.1) in $\mathbb{R}^{n}$ if and only if $v$, defined on $\mathbb{R}_{+}^{n+1}=$ $\left\{(x, \lambda): x \in \mathbb{R}^{n}, \lambda>0\right\}$, is a solution of the problem

$$
\begin{cases}\operatorname{div}\left(\lambda^{1-2 s} \nabla v\right)=0 & \text { in } \mathbb{R}_{+}^{n+1} \\ -d_{s} \lim _{\lambda \rightarrow 0} \lambda^{1-2 s} \partial_{\lambda} v=f(v) & \text { on } \mathbb{R}^{n}\end{cases}
$$

where $v(x, 0)=u(x)$ on $\mathbb{R}^{n}=\partial \mathbb{R}_{+}^{n+1}$ and

$$
d_{s}=2^{2 s-1} \frac{\Gamma(s)}{\Gamma(1-s)}
$$

is a positive constant. The fact that this constant does not depend on $n$ is already shown in section 3.2 of [9]. Its precise value has been computed in several papers; see, e.g., [15, 29]. Using that $s \Gamma(s)=\Gamma(s+1)$ and $(1-s) \Gamma(1-s)=\Gamma(2-s)$ we deduce, respectively, that

$$
\frac{d_{s}}{(2 s)^{-1}} \rightarrow 1 \text { as } s \rightarrow 0 \text { and } \frac{d_{s}}{2(1-s)} \rightarrow 1 \text { as } s \rightarrow 1
$$

Thus, $d_{s}$ blows-up as $s \rightarrow 0$ and $d_{s}$ tends linearly to zero as $s \rightarrow 1$.

In the sequel, the extension $v$ of $u$ in $\mathbb{R}_{+}^{n+1}$ which satisfies $\operatorname{div}\left(\lambda^{1-2 s} \nabla v\right)=0$ will be named "the s-extension of $u$ ".

Observe that for every $0<s<1$, we have that $-1<1-2 s<1$ and thus the weight $\lambda^{1-2 s}$ which appears in (1.2) belongs to the Muckenhoupt class $A_{2}$. As a consequence, the theory developed by Fabes, Kenig, and Serapioni [14] applies to problem (1.2) and thus a Poincaré inequality, a Harnack inequality, and Hölder regularity hold for solutions of our problem. As shown by the first author and Sire [5], solutions to (1.2) are $C^{\beta}\left(\overline{\mathbb{R}_{+}^{n+1}}\right)$ for some $\beta \in(0,1)$. But in general they have no further regularity in $\lambda$; note that $\lambda^{2 s}$ (even that it is unbounded) solves (1.2) with $f$ identically constant. The trace $u(\cdot, 0)$ on $\{\lambda=0\}$ of a bounded solution of (1.2) is however a $C^{2, \beta}\left(\mathbb{R}^{n}\right)$ function; see [5]. We will also use some gradient estimates from [5] which apply to 
solutions of (1.2) (see Remark 1.10 below) and that will be important in the proof of our energy estimates.

Problem (1.2) associated to the nonlocal equation (1.1) allows to introduce a notion of energy and global minimality for a solution $u$ of problem (1.1).

Consider the cylinder

$$
C_{R}=B_{R} \times(0, R) \subset \mathbb{R}_{+}^{n+1},
$$

where $B_{R}$ is the ball of radius $R$ centered at 0 in $\mathbb{R}^{n}$, and the energy functional

$$
\mathcal{E}_{s, C_{R}}(v)=d_{s} \int_{C_{R}} \frac{1}{2} \lambda^{1-2 s}|\nabla v|^{2} d x d \lambda+\int_{B_{R}} G(v(x, 0)) d x
$$

where $G^{\prime}=-f$ and thus $G$ is defined up to an additive constant. Observe that $\mathcal{E}_{s, C_{R}}$ is the energy functional associated to problem (1.2). We can now give the following definition. Let us denote, for $\Omega \subset \mathbb{R}_{+}^{n+1}$, the Sobolev space $H^{1}\left(\Omega, \lambda^{1-2 s}\right)$ made of functions $v$ with $\lambda^{1-2 s}\left(v^{2}+|\nabla v|^{2}\right) \in L^{1}(\Omega)$.

Definition 1.1. a) We say that a bounded $C_{\mathrm{loc}}^{\beta}\left(\overline{\mathbb{R}_{+}^{n+1}}\right) \cap H_{\mathrm{loc}}^{1}\left(\overline{\mathbb{R}_{+}^{n+1}}, \lambda^{1-2 s}\right)$ function $v$ is a global minimizer of (1.2) if, for all $R>0$,

$$
\mathcal{E}_{s, C_{R}}(v) \leq \mathcal{E}_{s, C_{R}}(w),
$$

for every $H^{1}\left(C_{R}, \lambda^{1-2 s}\right)$ function $w$ such that $v \equiv w$ in $\partial C_{R} \backslash\{\lambda=0\}$.

b) We say that a bounded $C^{1}$ function $u$ in $\mathbb{R}^{n}$ is a global minimizer of (1.1) if its $s$-extension $v$ is a global minimizer of (1.2).

c) We say that a bounded function $u$ is a layer solution of (1.1) if $u$ is a solution, it is monotone increasing in one of the $x$-variables, say $\partial_{x_{n}} u>0$ in $\mathbb{R}^{n}$, and

$$
\lim _{x_{n} \rightarrow \pm \infty} u\left(x^{\prime}, x_{n}\right)= \pm 1 \quad \text { for every } x^{\prime} \in \mathbb{R}^{n-1} .
$$

We recall that every layer solution is a global minimizer (see [6]). This is proved using the sliding method. Note that the weight $\lambda^{1-2 s}$ does not depend on the horizontal variables $x_{1}, \ldots, x_{n}$, and hence problem (1.2) is invariant under translations in the directions $x_{1}, \ldots, x_{n}$.

Our first result is the following energy estimate for global minimizers of problem (1.1). In particular it applies also to layer solutions. Given a nonlinearity $f$ and a bounded function $u$ defined on $\mathbb{R}^{n}$, set

$$
\begin{gathered}
G(u)=\int_{u}^{1} f \text { and } \\
c_{u}=\min \left\{G(s): \inf _{\mathbb{R}^{n}} u \leq s \leq \sup _{\mathbb{R}^{n}} u\right\} .
\end{gathered}
$$


Theorem 1.2. Let $s \in(0,1), f$ be any $C^{1, \gamma}$ nonlinearity with $\gamma>\max \{0,1-2 s\}$, and let $u: \mathbb{R}^{n} \rightarrow \mathbb{R}$ be a bounded global minimizer of (1.1). Let $v$ be the s-extension of $u$ in $\mathbb{R}_{+}^{n+1}$.

Then, for all $R>2$,

$$
\mathcal{E}_{s, C_{R}}(v)=d_{s} \int_{C_{R}} \frac{1}{2} \lambda^{1-2 s}|\nabla v|^{2} d x d \lambda+\int_{B_{R}}\left\{G(u)-c_{u}\right\} d x \leq C R^{n-2 s} \int_{1 / R}^{1} \rho^{-2 s} d \rho,
$$

where $c_{u}$ is defined by (1.5), and $C$ is a positive constant depending only on $n, s$, $\|f\|_{C^{1, \gamma}\left(\left[\inf _{\mathbb{R}^{n}} u, \sup _{\mathbb{R}^{n}} u\right]\right)}$, and $\|u\|_{L^{\infty}\left(\mathbb{R}^{n}\right)}$.

As a consequence, for some constant $C$ depending on the same quantities as before, we have

$$
\begin{aligned}
& \mathcal{E}_{s, C_{R}}(v) \leq C R^{n-2 s} \quad \text { if } 0<s<1 / 2, \\
& \mathcal{E}_{s, C_{R}}(v) \leq C R^{n-1} \log R \quad \text { if } s=1 / 2, \\
& \mathcal{E}_{s, C_{R}}(v) \leq C R^{n-1} \quad \text { if } 1 / 2<s<1 .
\end{aligned}
$$

For $s=1 / 2$, the estimate was proved in our previous paper [4]. For $s \in(0,1)$ they were announced in the second author Ph.D. Thesis [12]. More recently the estimates have been proven with a different method (without using the extension problem) by Savin and Valdinoci [24]. While their proof is simpler than ours, we develope further results which are of independent interest. More precisely, an extension result (Theorem [1.7) and a phase transition estimate (Theorem [1.9) both involving fractional Sobolev norms with weights.

Remark 1.3. The energy estimate (1.7) is sharp. Indeed, for every bounded solution of problem (1.1) the energy is also bounded below by $c_{1} R^{n-2 s}$ for some constant $c_{1}>0$. This is a consequence of a monotonicity formula (Proposition 3.2) that we prove in section 3 .

Moreover, also when $1 / 2 \leq s<1$ the energy estimates (1.8) and (1.9) are sharp since they are optimal for 1-D layer solutions, in the sense that for these solutions the energy is also bounded below by $c_{2} R^{n-1} \log R$ (if $s=1 / 2$ ) and $c_{2} R^{n-1}$ (if $s>1 / 2$ ), for some constant $c_{2}>0$, when they are seen as solutions in $\mathbb{R}^{n}$. When $s>1 / 2$, this follows immediately from Fubini's theorem. Indeed, if $v=v\left(x_{n}, \lambda\right)$ is a 1-D layer 
solution of (1.2) then, for $R>1$ we have (note that $G(u)-c_{u} \geq 0$ in $\mathbb{R}^{n}$ )

$$
\begin{aligned}
\mathcal{E}_{s, C_{\sqrt{n} R}}(v) \geq & \mathcal{E}_{s,(-R, R)^{n} \times(0, R)}(v)=d_{s} \int_{(-R, R)^{n} \times(0, R)} \frac{1}{2} \lambda^{1-2 s}\left|\nabla v\left(x_{n}, \lambda\right)\right|^{2} d x d \lambda+ \\
& +\int_{(-R, R)^{n}}\left\{G\left(v\left(x_{n}, 0\right)\right)-c_{u}\right\} d x \\
= & R^{n-1}\left\{d_{s} \int_{0}^{R} d \lambda \int_{-R}^{R} \frac{1}{2} \lambda^{1-2 s}|\nabla v|^{2} d x_{n}+\int_{-R}^{R}\left\{G\left(v\left(x_{n}, 0\right)\right)-c_{u}\right\} d x_{n}\right\} \\
\geq & R^{n-1} d_{s} \int_{0}^{1} d \lambda \int_{-1}^{1} \frac{1}{2} \lambda^{1-2 s}\left|\nabla v\left(x_{n}, \lambda\right)\right|^{2} d x_{n}=C R^{n-1} .
\end{aligned}
$$

In our next result we establish that in dimension $n=3$ the energy estimate (1.6) holds also for bounded monotone solutions without the limit assumption (1.4). These solutions can only be guaranteed to be minimizers among a certain class of functions (see Proposition 6.2), but could fail to be global minimizers as defined before.

Theorem 1.4. Let $n=3, f$ be any $C^{1, \gamma}$ nonlinearity with $\gamma>\max \{0,1-2 s\}$ and $u$ be a bounded solution of (1.1) such that $\partial_{e} u>0$ in $\mathbb{R}^{3}$ for some direction $e \in \mathbb{R}^{3}$, $|e|=1$. Let $v$ be the s-extension of $u$ in $\mathbb{R}_{+}^{4}$.

Then, $v$ satisfies the energy estimate (1.6) with $n=3$.

In dimension $n=3$ and for every $1 / 2 \leq s<1$, Theorems 1.2 and 1.4 lead to the 1-D symmetry of bounded global minimizers and of bounded monotone solutions of problem (1.1). For $s=1 / 2$ this was proved in our previous paper [4].

Theorem 1.5. Assume that $n=3$ and $1 / 2 \leq s<1$. Let $f$ be any $C^{1, \gamma}$ nonlinearity with $\gamma>\max \{0,1-2 s\}$ and $u$ be either a bounded global minimizer of (1.1), or a bounded solution of (1.1) which is monotone in some direction $e \in \mathbb{R}^{3},|e|=1$, i.e., it satisfies $\partial_{e} u>0$ in $\mathbb{R}^{3}$.

Then, $u$ depends only on one variable, i.e., there exists $a \in \mathbb{R}^{3}$ and $g: \mathbb{R} \rightarrow \mathbb{R}$, such that $u(x)=g(a \cdot x)$ for all $x \in \mathbb{R}^{3}$. Equivalently, the level sets of $u$ are planes.

Remark 1.6. In [8] Caffarelli, Roquejoffre, and Savin introduced and developed a regularity theory for nonlocal minimal surfaces. These surfaces, defined for $0<s<$ $1 / 2$, can be interpreted as a non-infinitesimal version of classical minimal surfaces and arise when minimizing in an appropriate way the $H^{s}$-norm of the indicator function. Note that when $0<s<1 / 2$ the indicator functions belong to $H^{s}$ and the extension problem (1.2) is a well posed problem for indicator functions. The authors also proved a sharp energy estimate $C R^{n-2 s}$ related to ours in some sense: our equation is the Allen-Cahn approximation of these nonlocal minimal surfaces. The flatness of any 
$s$-minimal surface in all $\mathbb{R}^{n}$ is only known in dimension $n=2$ by a recent result of Savin and Valdinoci [26] (this is the analogue statement to that of the conjecture of De Giorgi). The same statement for $s$ sufficiently close to $1 / 2$ and $n \leq 7$ had been proved by Caffarelli and Valdinoci [11].

To prove 1-D symmetry, we use a Liouville type argument which requires an appropriate estimate for the Dirichlet energy. By a result of Moschini [19], if the energy estimate

$$
\int_{C_{R}}|\nabla v|^{2} d x d \lambda \leq C R^{2} \log R
$$

holds (note the exponent 2 in $R^{2} \log R$ ), then one may use such Liouville type result and deduce 1-D symmetry in $\mathbb{R}^{n}$ for global minimizers and for solutions which are monotone in one direction. Now, by Theorems 1.2 and 1.4, we have that

$$
\int_{C_{R}} \frac{1}{2} \lambda^{1-2 s}|\nabla v|^{2} d x d \lambda \leq C R^{2} \log R
$$

holds for $n=3$ and every $1 / 2 \leq s<1$. Instead, if $0<s<1 / 2$, the sharp estimate is

$$
\int_{C_{R}} \frac{1}{2} \lambda^{1-2 s}|\nabla v|^{2} d x d \lambda \leq C R^{3-2 s} .
$$

Since $3-2 s>2$ when $0<s<1 / 2$, one can not use the Liouville argument. This is the reason why we can prove 1 -D symmetry only for $n \leq 3$, and in case $n=3$ only for $1 / 2 \leq s<1$.

We have two different proofs of our energy estimate (1.6).

The first one is simple but applies only to bistable nonlinearities (such as the Allen-Cahn nonlinearity $f(u)=u-u^{3}$ ) and to monotone solutions satisfying the limit assumption (1.4) or the more general (2.3) below. We present this very simple proof in section 2. It was found by Ambrosio and the first author [3] to prove the optimal energy estimate for $-\Delta u=u-u^{3}$ in $\mathbb{R}^{n}$. In this specific case of bistable nonlinearities and monotone solutions satisfying (2.3), our energy estimate is uniform as $s \uparrow 1$. On the contrary, the energy estimate for global minimizers and general $f$ (as stated in Theorem 1.2) is not uniform as $s \uparrow 1$. Instead, the estimate in [24] is uniform as $s \uparrow 1$, even if not stated in that paper.

Our second proof applies in more general situations and will lead to Theorems 1.2 and 1.4. It is based on controlling the weighted $H^{1}\left(C_{R}, \lambda^{1-2 s}\right)$-norm of the solution $v$ by certain weighted fractional Sobolev norms of the trace of $v$ on $\partial C_{R}$.

Let us recall the definition of the $H^{s}(A)$-norm of a function for $0<s<1$, where $A$ is either a bounded Lipschitz domain of $\mathbb{R}^{n}$, or $A=\partial \Omega$ and $\Omega$ is a bounded Lipschitz 
domain of $\mathbb{R}^{n+1}$. It is given by

$$
\|w\|_{H^{s}(A)}^{2}=\|w\|_{L^{2}(A)}^{2}+\int_{A} \int_{A} \frac{|w(z)-w(\bar{z})|^{2}}{|z-\bar{z}|^{n+2 s}} d \mathcal{H}^{n}(z) d \mathcal{H}^{n}(\bar{z}),
$$

where $d \mathcal{H}^{n}$ denotes the $n$-dimensional Hausdorff measure. In the sequel we will use it for $\Omega=C_{1}=B_{1} \times(0,1) \subset \mathbb{R}^{n+1}$ and $A=\partial C_{1}$.

To prove Theorem 1.2, we use the following comparison argument. We construct a comparison function $\bar{w}$ which takes the same values of $v$ on $\partial C_{R} \cap\{\lambda>0\}$ and thus, by minimality of $v$,

$$
\mathcal{E}_{C_{R}}(v) \leq \mathcal{E}_{C_{R}}(\bar{w})
$$

Then, it is enough to estimate the energy of $\bar{w}$.

For simplicity, consider the case of the Allen-Cahn nonlinearity. We define the function $\bar{w}(x, \lambda)$ in $C_{R}$ in the following way. First we define $\bar{w}(x, 0)$ on the base of the cylinder as a smooth function $g(x)$ which is identically equal to 1 in $B_{R-1}$ and $g(x)=v(x, 0)$ for $|x|=R$; then we define $\bar{w}(x, \lambda)$ as the unique solution of the Dirichlet problem

$$
\begin{cases}\operatorname{div}\left(\lambda^{1-2 s} \nabla \bar{w}\right)=0 & \text { in } C_{R} \\ \bar{w}(x, 0)=g(x) & \text { on } B_{R} \times\{\lambda=0\} \\ \bar{w}(x, \lambda)=v(x, \lambda) & \text { on } \partial C_{R} \cap\{\lambda>0\}\end{cases}
$$

Since by definition $\bar{w} \equiv 1$ on $B_{R-1} \times\{0\}$, then the potential energy is bounded by $C\left|B_{R} \backslash B_{R-1}\right|=C R^{n-1}$. Thus, it remains to estimate the Dirichlet energy.

To do this we proceed in two steps. First, after rescaling, we apply Theorem 1.7 below, to control the Dirichlet norm of $\bar{w}_{1}$ (where $\bar{w}_{1}$ is the rescaled version of $\bar{w}$ ) in $C_{1}$ by some fractional Sobolev norms of its trace on $\partial C_{1}$. Then, we use Theorem 1.9 below to give an estimate of these fractional norms.

We recall that in the proof of the estimate for the Dirichlet energy for $s=1 / 2$ a crucial point was an extension theorem which let us control the $H^{1}\left(C_{1}\right)$-norm of a function by the $H^{1 / 2}\left(\partial C_{1}\right)$-norm of its trace. Here we are in a more complicated situation, since we need to control the weighted $H^{1}\left(C_{1}, \lambda^{1-2 s}\right)$-norm, with a weight which degenerates or blows-up on a subset of $\partial C_{1}$ (its bottom).

We introduce some notation. Let $A$ be either a bounded Lipschitz domain in $\mathbb{R}^{n}$ or $A=\partial \Omega$ where $\Omega$ is a bounded domain of $\mathbb{R}^{n+1}$ with Lipschitz boundary. Let $M \subset A$ be an open set (relative to $A$ ) with Lipschitz boundary (relative to $A$ ) $\Gamma=\partial M$. We 
define the following two sets:

$$
B_{\text {frac }}= \begin{cases}A \times A & \text { if } 0<s \leq 1 / 2 \\ M \times M & \text { if } 1 / 2<s<1\end{cases}
$$

and

$$
B_{\text {weig }}= \begin{cases}(A \backslash M) \times(A \backslash M) & \text { if } 0<s \leq 1 / 2 \\ (A \backslash M) \times A & \text { if } 1 / 2<s<1 .\end{cases}
$$

For every $z$, we denote $d_{M}(z)$ the Euclidean distance (either in $\mathbb{R}^{n}$ or in $\mathbb{R}^{n+1}$ ) from the point $z$ to the set $M$. Set

$$
a:=1-2 s \in(-1,1) .
$$

In Theorem 1.7 we establish that, for any given function $w$ defined on all $\partial \Omega$, there exists an extension $\widetilde{w}$ of $w$ to $\Omega$ whose $H^{1}\left(\Omega, d_{M}^{a}\right)$-norm is controlled by a combination of a $H^{s}$-norm and a $H^{1 / 2}\left(\cdot, d_{M}^{a}\right)$-norm of its trace $w$. If $h$ is a weight (that is, a nonnegative function here), we indicate with $H^{s}(\partial \Omega, h)$ the weighted Sobolev space of functions $w$ such that

$$
\int_{\partial \Omega} h(z) w(z)^{2} d \mathcal{H}^{n}(z)+\int_{\partial \Omega} \int_{\partial \Omega} h(z) \frac{|w(z)-w(\bar{z})|^{2}}{|z-\bar{z}|^{n+2 s}} d \mathcal{H}^{n}(z) d \mathcal{H}^{n}(\bar{z})<+\infty .
$$

Later we will apply our results in the case

$\Omega=C_{1}, A=\partial C_{1}, M=B_{1} \times\{0\}$, and $h=d_{M}^{1-2 s}(x, \lambda)=\operatorname{dist}^{1-2 s}((x, \lambda), M)=\lambda^{1-2 s}$.

For a general domain the result is the following.

Theorem 1.7. Let $\Omega$ be a bounded domain of $\mathbb{R}^{n+1}$ with Lipschitz boundary $\partial \Omega$ and $M \subset \partial \Omega$ an open subset (relative to $\partial \Omega$ ) with Lipschitz boundary (relative to $\partial \Omega$ ). For $z \in \mathbb{R}^{n+1}$, let $d_{M}(z)$ denote the Euclidean distance from the point $z$ to the set $M$. Let $s \in(0,1)$.

Then, for every $w \in C(\partial \Omega)$ there exists an extension $\widetilde{w}$ of $w$ defined in $\bar{\Omega}$ which belongs to $C^{1}(\Omega) \cap C(\bar{\Omega})$ and such that

$$
\begin{aligned}
\int_{\Omega} d_{M}(z)^{1-2 s}|\nabla \widetilde{w}|^{2} d z & \\
\leq & \left.C|| w\right|_{L^{2}(\partial \Omega)} ^{2}+C \iint_{B_{\mathrm{frac}}} \frac{|w(z)-w(\bar{z})|^{2}}{|z-\bar{z}|^{n+2 s}} d \mathcal{H}^{n}(z) d \mathcal{H}^{n}(\bar{z}) \\
& +C \iint_{B_{\text {weig }}} d_{M}(z)^{1-2 s} \frac{|w(z)-w(\bar{z})|^{2}}{|z-\bar{z}|^{n+1}} d \mathcal{H}^{n}(z) d \mathcal{H}^{n}(\bar{z}),
\end{aligned}
$$


where $B_{\text {frac }}$ and $B_{\text {weig }}$ are defined, respectively, in (1.11) and (1.12) with $A=\partial \Omega$, and $C$ denotes a positive constant depending only on $\Omega, M$, and $s$.

We have used the notations $B_{\text {frac }}$ and $B_{\text {weig }}$ to indicate, respectively, the set in which we compute the $H^{s}$-norm of $w$ and the set in which we compute the weighted $H^{1 / 2}\left(\cdot, d_{M}^{a}\right)$-norm of $w$.

Remark 1.8. We denote by $\bar{w}$ the $s$-extension of $w$ in $\Omega$. Since $\bar{w}$ is the extension of $w$ in $\Omega$ which minimizes the quantity

$$
\int_{\Omega} d_{M}(z)^{1-2 s}|\nabla \widetilde{w}|^{2} d z
$$

then inequality (1.13) holds, in particular, with $\widetilde{w}$ replaced by $\bar{w}$.

In two articles [20, 21], Nekvinda treated some extension and trace problems for functions belonging to fractional Sobolev spaces, but his results are not applicable in our situation. In [21], the author proved an extension theorem for functions belonging to $H^{s}(M)$, where $M$ is, as before, a subset of $\partial \Omega$. More precisely he proved that if $w \in H^{s}(M)$ then there exists an extension $\widetilde{w}$ of $w$ in $\Omega$ such that

$$
\int_{\Omega} d_{M}(z)^{1-2 s}|\nabla \widetilde{w}|^{2} d z \leq C\|w\|_{H^{s}(M)}^{2}
$$

Notice that this theorem gives an extension for a function defined only on $M$. There is no control on the extension near $\partial \Omega \backslash M$ - a control that we require. Instead, in [20], he considered the case of a function $w$ defined on $\partial \Omega \backslash M$ and established that there exists an extension $\widetilde{w}$ of $w$ in $\Omega$ with $H^{1}\left(\cdot, d_{M}^{a}\right)$-norm controlled by some weighted fractional norm of $w$ in $\partial \Omega \backslash M$. In our situation, we need an extension result to all of $\Omega$ for functions $w$ defined on all of $\partial \Omega$.

We conclude giving a key result in the proof of Theorem 1.2 on energy estimates for minimizers. It will give control on the $H^{s}$ double integrals above on $A:=\partial \Omega$ knowing the following assumptions on the function $w$ defined on $A$.

Let $A, M \subset A, \Gamma=\partial M, B_{\text {frac }}$, and $B_{\text {weig }}$ be as in (1.11) and (1.12). Let $D$ denote all tangential derivatives to $A$ and $d_{\Gamma}(z)$ denote the Euclidean distance from the point $z$ to the set $\Gamma$ (either in $\mathbb{R}^{n}$ or in $\mathbb{R}^{n+1}$ ). Note that here we deal with the distance $d_{\Gamma}=d_{\partial M}$ to $\Gamma=\partial M$, in contrast with the distance $d_{M}$ to $M$ appearing in the weighted energies (1.13) above and (1.17) below.

In what follows we will assume that, for some constant $c_{s}, w$ satisfies these conditions: 
- for $s \in(0,1 / 2]$,

$$
|D w(z)| \leq \begin{cases}\frac{c_{s}}{\varepsilon}\left(\frac{d_{\Gamma}(z)}{\varepsilon}\right)^{2 s-1} & \text { if } z \in A \text { and } d_{\Gamma}(z) \leq \varepsilon \\ \frac{c_{s}}{d_{\Gamma}(z)} & \text { if } z \in A \text { and } d_{\Gamma}(z)>\varepsilon\end{cases}
$$

- for $s \in(1 / 2,1)$,

$$
|D w(z)| \leq \begin{cases}\frac{c_{s}}{\varepsilon} & \text { if } z \in A \text { and } d_{\Gamma}(z) \leq \varepsilon \\ \frac{c_{s}}{d_{\Gamma}(z)} & \text { if } z \in A \text { and } d_{\Gamma}(z)>\varepsilon\end{cases}
$$

Later we will use this result with

$$
A=\partial C_{1}, M=B_{1} \times\{\lambda=0\}, \text { and } \Gamma=\partial B_{1} \times\{\lambda=0\} .
$$

In more general geometries the result is the following.

Theorem 1.9. Let $A$ be either a bounded Lipschitz domain in $\mathbb{R}^{n}$ or $A=\partial \Omega$ where $\Omega$ is a bounded domain of $\mathbb{R}^{n+1}$ with Lipschitz boundary. Let $M \subset A$ be an open set (relative to $A$ ) with Lipschitz boundary (relative to $A) \Gamma=\partial M$. Let $\varepsilon \in(0,1 / 2)$ and $s \in(0,1)$.

Suppose that, for some constant $c_{s}, w: A \rightarrow \mathbb{R}$ is a Lipschitz function such that

$$
|w(z)| \leq c_{s}
$$

and that $w$ satisfies (1.14) and (1.15) for almost every $z \in A$.

Then,

$$
\begin{aligned}
& \Psi_{s}(w):=\|w\|_{L^{2}(A)}^{2}+\iint_{B_{\mathrm{frac}}} \frac{|w(z)-w(\bar{z})|^{2}}{|z-\bar{z}|^{n+2 s}} d \mathcal{H}^{n}(z) d \mathcal{H}^{n}(\bar{z}) \\
& +\iint_{B_{\mathrm{weig}}} d_{M}(z)^{1-2 s} \frac{|w(z)-w(\bar{z})|^{2}}{|z-\bar{z}|^{n+1}} d \mathcal{H}^{n}(z) d \mathcal{H}^{n}(\bar{z}) \leq C \int_{\varepsilon}^{1} \rho^{-2 s} d \rho,
\end{aligned}
$$

where the sets $B_{\text {frac }}$ and $B_{\text {weig }}$ are defined in (1.11) and (1.12), and $C$ denotes a positive constant depending on $A, M, n, s$, and $c_{s}$.

As a consequence, for some constant $C$ depending on the same quantities as before, we have

$$
\Psi_{s}(w) \leq \begin{cases}C & \text { if } 0<s<1 / 2 \\ C|\log \varepsilon| & \text { if } s=1 / 2 \\ C \varepsilon^{1-2 s} & \text { if } 1 / 2<s<1\end{cases}
$$

In our case $\left(A=\partial C_{1}, M=B_{1} \times\{\lambda=0\}, \Gamma=\partial B_{1} \times\{\lambda=0\}\right)$, the constant $C$ in (1.17) only depends on $n, s$, and $c_{s}$. 
Remark 1.10. In the proof of Theorem 1.2 the following gradient estimates for every bounded solution $v$ of problem (1.2) will be of utmost importance. Let $f \in C^{1, \gamma}$ for some $\gamma>\max \{0,1-2 s\}$. Then, every bounded solution $v$ of (1.2) satisfies, for some constant $c_{s}$,

$$
\begin{cases}\left|\nabla_{x} v(x, \lambda)\right| \leq c_{s} & \text { for every } x \in \mathbb{R}^{n} \text { and } \lambda \geq 0 \\ |\nabla v(x, \lambda)| \leq c_{s} / \lambda & \text { for every } x \in \mathbb{R}^{n} \text { and } \lambda>0 \\ \left|\lambda^{1-2 s} \partial_{\lambda} v\right| \leq c_{s} \quad \text { for every } x \in \mathbb{R}^{n} \text { and } \lambda>0\end{cases}
$$

For the bound $\left|\nabla_{x} v(x, 0)\right| \leq c_{s}$ for $x \in \mathbb{R}^{n}$, see Silvestre [27], Lemmas 2.8 and 2.9 in [27]. In this inequality the constant $c_{s}$ is uniformly bounded for $s$ away from zero, but not as $s \rightarrow 0$. For this reason the constant $C$ in our energy estimate (1.6) is not uniform for $s$ close to zero. Using the maximum principle we can extend the bound $\left|\nabla_{x} v(x, 0)\right| \leq c_{s}$ to every $\lambda>0$ and deduce $\left|\nabla_{x} v(x, \lambda)\right| \leq c_{s}$ for every $x \in \mathbb{R}^{n}$ and $\lambda \geq 0$; see Proposition 4.6 of $[5]$.

The bound $|\nabla v(x, \lambda)| \leq c_{s} / \lambda$ for every $x \in \mathbb{R}^{n}$ and $\lambda>0$ follows, after rescaling, by interior elliptic estimates, since equation (1.2) is uniformly elliptic for $\lambda>0$; see Proposition 4.6 of [5]. In this bound the constant $c_{s}$ is also bounded as $s \uparrow 1$.

Finally, the last bound $\left|\lambda^{1-2 s} \partial_{\lambda} v(x, \lambda)\right| \leq c_{s}$ for every $x \in \mathbb{R}^{n}$ and $\lambda \geq 0$ is established using that the function $\widetilde{v}=\lambda^{1-2 s} \partial_{\lambda} v$ satisfies the dual problem (with Dirichlet boundary condition)

$$
\begin{cases}\operatorname{div}\left(\lambda^{2 s-1} \nabla \widetilde{v}\right)=0 & \text { in } \mathbb{R}_{+}^{n+1} \\ \widetilde{v}=-\frac{f(u)}{d_{s}} & \text { on } \partial \mathbb{R}_{+}^{n+1} ;\end{cases}
$$

see also Proposition 4.6 of [5]. In this last gradient estimate, the constant $c_{s}$ is uniformly bounded for $s$ away from 1 but not as $s \rightarrow 1$ (since $d_{s} /(1-s) \rightarrow 1$ as $s \rightarrow 1$ ). For this reason, the constant $C$ in our energy estimate (1.6) blows up for $s$ close to 1 .

The paper is organized as follows:

- In section 2 we prove the energy estimate for layer solutions of bistable type equations, using a simple argument introduced by Ambrosio and the first author 3.

- In section 3 we establish a monotonicity formula for the energy functional associated to problem (1.2).

- In section 4 we give the proof of the extension Theorem 1.7 and of the key Theorem 1.9.

- In section 5 we prove Theorem 1.2 . 
- In section 6 we establish energy estimates for bounded monotone solutions in $\mathbb{R}^{3}$ (Theorem 1.4 ).

- In section 7 we prove the $1-\mathrm{D}$ symmetry result, that is Theorem 1.5.

\section{EnERGy ESTimate For monotone solutions of Bistable EQUATIONS}

In this section we consider potentials $G(u)=\int_{u}^{1} f$ satisfying the following hypothesis:

$$
G \geq 0=G( \pm 1) \quad \text { in } \mathbb{R} \text { and } G>0 \text { in }(-1,1) .
$$

An example is $G(u)=\frac{1}{4}\left(1-u^{2}\right)^{2}$. In this case the nonlinearity is given by $f(u)=$ $u-u^{3}$. In [5, 6] it is proved the existence of a 1-D layer solution for every nonlinearity satisfying (2.1).

In the sequel we consider the energy

$$
\mathcal{E}_{s, C_{R}}(v)=d_{s} \int_{C_{R}} \frac{1}{2} \lambda^{1-2 s}|\nabla v|^{2} d x d \lambda+\int_{B_{R}} G(v(x, 0)) d x .
$$

In the following theorem we establish energy estimates for monotone solutions of (1.1) such that $\lim _{x_{n} \rightarrow+\infty} u\left(x^{\prime}, x_{n}\right)=1$ for all $x^{\prime} \in \mathbb{R}^{n-1}$, assuming that the potential $G$ satisfies (2.1). We point out that here the constant appearing in our energy estimate is uniformly bounded as $s \uparrow 1$ (but not as $s \downarrow 0$ ). Recall that we have defined the cylinder $C_{R}=B_{R} \times(0, R)$, where $B_{R}$ is the ball centered at 0 and of radius $R$ in $\mathbb{R}^{n}$.

Theorem 2.1. Let $f$ be a $C^{1, \gamma}$ function, with $\gamma>\max \{0,1-2 s\}$. Suppose that $G(u)=\int_{u}^{1} f$ satisfies (2.1). Let $u$ be a solution of problem (1.1) in $\mathbb{R}^{n}$, with $|u|<1$, and let $v$ be the s-extension of $u$ in $\mathbb{R}_{+}^{n+1}$.

Given any $s_{0} \in(0,1 / 2)$, assume that $s_{0}<s<1$.

Suppose that

$$
u_{x_{n}}>0 \text { in } \mathbb{R}^{n}
$$

and

$$
\lim _{x_{n} \rightarrow+\infty} u\left(x^{\prime}, x_{n}\right)=1 \text { for all } x^{\prime} \in \mathbb{R}^{n-1} .
$$

Then, $v$ satisfies the energy estimate (1.6) for every $R>2$, where the constant $C$ depends only on $n, s_{0}$, and $\|f\|_{C^{1, \gamma}([-1,1])}$. In particular, for some constant $C$ depending on these three quantities, we have

$$
\begin{gathered}
\mathcal{E}_{s, C_{R}}(v) \leq \frac{C}{1-2 s} R^{3-2 s} \quad \text { if } s_{0}<s<1 / 2 \\
\mathcal{E}_{s, C_{R}}(v) \leq C R^{2} \log R \quad \text { if } s=1 / 2 \\
\mathcal{E}_{s, C_{R}}(v) \leq \frac{C}{2 s-1} R^{2} \quad \text { if } 1 / 2<s<1 .
\end{gathered}
$$


Proof. As in [4], the proof uses an argument found by Ambrosio and the first author [3] to prove an energy estimate for layer solutions of the analogue problem $-\Delta u=f(u)$. This method is based on sliding the function $v$ in the direction $x_{n}$. Consider the function

$$
v^{t}(x, \lambda):=v\left(x^{\prime}, x_{n}+t, \lambda\right)
$$

defined for $(x, \lambda)=\left(x^{\prime}, x_{n}, \lambda\right) \in \mathbb{R}_{+}^{n+1}$ and $t \in \mathbb{R}$. For each $t$ we have

$$
\begin{cases}\operatorname{div}\left(\lambda^{1-2 s} \nabla v^{t}\right)=0 & \text { in } \mathbb{R}_{+}^{n+1}, \\ -d_{s} \lambda^{1-2 s} \partial_{\lambda} v^{t}=f\left(v^{t}\right) & \text { on } \mathbb{R}^{n}=\partial \mathbb{R}_{+}^{n+1}\end{cases}
$$

Now we use the first two gradient estimates in (1.18) for the solution $v$ of problem (1.2). We have that for every $t,\left|v^{t}\right|<1$ and

$$
\begin{aligned}
& \left|\nabla_{x} v^{t}(x, \lambda)\right| \leq c_{s} \text { for every } x \in \mathbb{R}^{n} \text { and } \lambda \geq 0, \\
& \left|\nabla v^{t}(x, \lambda)\right| \leq \frac{c_{s}}{\lambda} \text { for every } x \in \mathbb{R}^{n} \text { and } \lambda>0
\end{aligned}
$$

where the constant $c_{s}$ is uniformly bounded for $s$ away from 0 (see Remark 1.10). In fact, $c_{s}$ depends only on $n, s_{0}$, and $\|f\|_{C^{1, \gamma([-1,1])}}$ (see Proposition 4.6 of [5]). In addition (see Lemma 4.8 of [5])

$$
\lim _{t \rightarrow+\infty}\left\{\left|v^{t}(x, \lambda)-1\right|+\left|\nabla v^{t}(x, \lambda)\right|\right\}=0
$$

for all $x \in \mathbb{R}^{n}$ and all $\lambda \geq 0$.

Note that hypothesis (2.2) and the maximum principle lead to $v_{x_{n}}>0$ in $\mathbb{R}_{+}^{n+1}$. Thus, denoting the derivative of $v^{t}(x, \lambda)$ with respect to $t$ by $\partial_{t} v^{t}(x, \lambda)$, we have

$$
\partial_{t} v^{t}(x, \lambda)=v_{x_{n}}\left(x^{\prime}, x_{n}+t, \lambda\right)>0 \quad \text { for all } x \in \mathbb{R}^{n}, \lambda \geq 0
$$

By (2.10), we have that

$$
\lim _{t \rightarrow+\infty} \mathcal{E}_{s, C_{R}}\left(v^{t}\right)=0
$$

Next, we bound the derivative of $\mathcal{E}_{s, C_{R}}\left(v^{t}\right)$ with respect to $t$. Recall that we have set $a=1-2 s$. We use that $v^{t}$ is a bounded solution of problem (1.2), the bounds (2.8), (2.9) for the derivatives of $v^{t}$, and the crucial fact that $\partial_{t} v^{t}>0$. Let $\nu$ denote the exterior normal to the lateral boundary $\partial B_{R} \times(0, R)$ of the cylinder $C_{R}$. 
We have

$$
\begin{aligned}
\partial_{t} \mathcal{E}_{s, C_{R}}\left(v^{t}\right) & =d_{s} \int_{0}^{R} d \lambda \int_{B_{R}} d x \lambda^{a} \nabla v^{t} \cdot \nabla\left(\partial_{t} v^{t}\right)+\int_{B_{R}} d x G^{\prime}\left(v^{t}(x, 0)\right) \partial_{t} v^{t}(x, 0) \\
& =d_{s} \int_{0}^{R} d \lambda \int_{\partial B_{R}} d \mathcal{H}^{n-1} \lambda^{a} \frac{\partial v^{t}}{\partial \nu} \partial_{t} v^{t}+d_{s} \int_{B_{R} \times\{\lambda=R\}} d x \lambda^{a} \frac{\partial v^{t}}{\partial \lambda} \partial_{t} v^{t}(x, R) \\
& \geq-C d_{s} \int_{0}^{R} d \lambda \frac{\lambda^{a}}{1+\lambda} \int_{\partial B_{R}} d \mathcal{H}^{n-1} \partial_{t} v^{t}-C d_{s} R^{-2 s} \int_{B_{R} \times\{\lambda=R\}} d x \partial_{t} v^{t}(x, R),
\end{aligned}
$$

where in the last inequality we have used both gradient bounds (2.8) and (2.9) for the first term (since $\frac{\partial v^{t}}{\partial \nu}$ is a horizontal derivative) and the bound (2.9) for the second term. We recall that here $C$ is a constant uniformly bounded for $s$ away from 0 . Hence, for every $T>0$, we have

$$
\begin{aligned}
\mathcal{E}_{s, C_{R}}(v)= & \mathcal{E}_{s, C_{R}}\left(v^{T}\right)-\int_{0}^{T} \partial_{t} \mathcal{E}_{s, C_{R}}\left(v^{t}\right) d t \\
\leq & \mathcal{E}_{s, C_{R}}\left(v^{T}\right)+C d_{s} \int_{0}^{T} d t \int_{0}^{R} d \lambda \frac{\lambda^{a}}{1+\lambda} \int_{\partial B_{R}} d \mathcal{H}^{n-1} \partial_{t} v^{t} \\
& +C d_{s} R^{-2 s} \int_{0}^{T} d t \int_{B_{R} \times\{\lambda=R\}} d x \partial_{t} v^{t}(x, R) \\
= & \mathcal{E}_{s, C_{R}}\left(v^{T}\right)+C d_{s} \int_{\partial B_{R}} d \mathcal{H}^{n-1} \int_{0}^{R} d \lambda \frac{\lambda^{a}}{1+\lambda} \int_{0}^{T} d t \partial_{t} v^{t}(x, \lambda) \\
& +C d_{s} R^{-2 s} \int_{B_{R} \times\{\lambda=R\}} d x \int_{0}^{T} d t \partial_{t} v^{t}(x, R) \\
= & \mathcal{E}_{s, C_{R}}\left(v^{T}\right)+C d_{s} \int_{\partial B_{R}} d \mathcal{H}^{n-1} \int_{0}^{R} d \lambda \frac{\lambda^{a}}{1+\lambda}\left(v^{T}-v^{0}\right)(x, \lambda) \\
& \quad+C d_{s} R^{-2 s} \int_{B_{R} \times\{\lambda=R\}} d x\left(v^{T}-v^{0}\right)(x, R) \\
\leq & \mathcal{E}_{s, C_{R}}\left(v^{T}\right)+C d_{s} R^{n-1} \int_{0}^{R} \frac{\lambda^{a}}{1+\lambda} d \lambda+C d_{s} R^{n-2 s} \\
\leq & \mathcal{E}_{s, C_{R}}\left(v^{T}\right)+C d_{s} R^{n-1}\left(\int_{0}^{1} \lambda^{1-2 s} d \lambda+\int_{1}^{R} \lambda^{-2 s} d \lambda\right)+C d_{s} R^{n-2 s} .
\end{aligned}
$$

Using the change of variables $\lambda=\rho R$, we have $\int_{0}^{1} \lambda^{1-2 s} d \lambda+\int_{1}^{R} \lambda^{-2 s} d \lambda=\frac{1}{2(1-s)}+$ $R^{1-2 s} \int_{1 / R}^{1} \rho^{-2 s} d \rho$. Thus, using that $d_{s} \approx 2(1-s)$ as $s \uparrow 1$ and distinguishing the 
three cases $s_{0}<s<1 / 2, s=1 / 2$, and $1 / 2<s<1$, we conclude

$$
\begin{aligned}
\mathcal{E}_{s, C_{R}}(v) & \leq \mathcal{E}_{s, C_{R}}\left(v^{T}\right)+C R^{n-1}+C(1-s) R^{n-2 s} \int_{1 / R}^{1} \rho^{-2 s} d \rho+C(1-s) R^{n-2 s} \\
& \leq \mathcal{E}_{s, C_{R}}\left(v^{T}\right)+C R^{n-2 s} \int_{1 / R}^{1} \rho^{-2 s} d \rho .
\end{aligned}
$$

Letting $T \rightarrow+\infty$ and using (2.11), we obtain the desired estimate.

\section{Monotonicity Formula}

In this section we establish a monotonicity formula for the energy functional associated to problem (1.2). More precisely, we prove that for every solution $v$ of problem (1.2) the quantity $\mathcal{E}_{\widetilde{B}_{R}^{+}}(v) / R^{n-2 s}$ is nondecreasing in $R$, where $\widetilde{B}_{R}^{+}$is the positive half ball in $\mathbb{R}_{+}^{n+1}$ centered at 0 and of radius $R$. From this result we deduce that our energy estimate (1.7) is sharp when $0<s<1 / 2$ (see Remark 3.3)

In the following lemma we prove a Pohozaev identity for solutions of problem (1.2) which will be important in the proof of our monotonicity formula. We use the following notation:

$$
\widetilde{B}_{R}^{+}=\left\{(x, \lambda) \in \mathbb{R}_{+}^{n+1}:|(x, \lambda)|<R\right\} \text { and } \partial^{+} \widetilde{B}_{R}^{+}=\partial \widetilde{B}_{R}^{+} \cap\{\lambda>0\} .
$$

Lemma 3.1. Let $s \in(0,1), f$ be any $C^{1, \gamma}$ nonlinearity with $\gamma>\max \{0,1-2 s\}$, and suppose that $v$ is a bounded solution of problem (1.2).

Then, for every $R>0$

$$
\begin{aligned}
& \frac{n-2 s}{2} \int_{\widetilde{B}_{R}^{+}} \lambda^{1-2 s}|\nabla v|^{2} d x d \lambda+n \int_{B_{R} \times\{0\}} d_{s}^{-1} G(v) d x= \\
& =\frac{R}{2} \int_{\partial^{+} \widetilde{B}_{R}^{+}} \lambda^{1-2 s}|\nabla v|^{2} d \mathcal{H}^{n}-R \int_{\partial^{+} \widetilde{B}_{R}^{+}} \lambda^{1-2 s}\left(\frac{\partial v}{\partial \nu}\right)^{2} d \mathcal{H}^{n}+ \\
& \quad+R \int_{\partial B_{R} \times\{0\}} d_{s}^{-1} G(v) d \mathcal{H}^{n-1},
\end{aligned}
$$

where $d \mathcal{H}^{n-1}$ and $d \mathcal{H}^{n}$ denote respectively the $(n-1)$-dimensional and $n$-dimensional Hausdorff measures and $\frac{\partial v}{\partial \nu}$ denotes the outer normal derivative of $v$ on $\partial^{+} \widetilde{B}_{R}^{+}$.

Proof. Set $z=(x, \lambda)$. Multiplying the equation $\operatorname{div}\left(\lambda^{1-2 s} \nabla v\right)=0$ by $\langle z, \nabla v\rangle=$ $\langle(x, \lambda), \nabla v\rangle$, we have

$$
\begin{aligned}
0 & =\operatorname{div}\left(\lambda^{1-2 s} \nabla v\right)\langle z, \nabla v\rangle \\
& =\operatorname{div}\left(\lambda^{1-2 s} \nabla v\langle z, \nabla v\rangle\right)-\lambda^{1-2 s}\left\{|\nabla v|^{2}+\left\langle z, \nabla\left(\frac{|\nabla v|^{2}}{2}\right)\right\rangle\right\} .
\end{aligned}
$$


Now, observe that

$$
\begin{aligned}
\lambda^{1-2 s}\left\langle z, \nabla\left(\frac{|\nabla v|^{2}}{2}\right)\right\rangle= & \operatorname{div}\left\{\lambda^{1-2 s} z\left(\frac{|\nabla v|^{2}}{2}\right)\right\} \\
& -\lambda^{1-2 s}(n+1) \frac{|\nabla v|^{2}}{2}-(1-2 s) \lambda^{1-2 s} \frac{|\nabla v|^{2}}{2} \\
= & \operatorname{div}\left\{\lambda^{1-2 s} z\left(\frac{|\nabla v|^{2}}{2}\right)\right\}-\frac{n+2-2 s}{2} \lambda^{1-2 s}|\nabla v|^{2}
\end{aligned}
$$

Thus, we obtain

$$
\operatorname{div}\left\{\lambda^{1-2 s}\left(\nabla v\langle z, \nabla v\rangle-z \frac{|\nabla v|^{2}}{2}\right)\right\}+\frac{n-2 s}{2} \lambda^{1-2 s}|\nabla v|^{2}=0 .
$$

Next, we integrate by parts on $\widetilde{B}_{R}^{+}$:

$$
\begin{aligned}
& \int_{\partial^{+} \widetilde{B}_{R}^{+}} \lambda^{1-2 s}\langle\nu, \nabla v\rangle\langle z, \nabla v\rangle d \mathcal{H}^{n}+\int_{B_{R} \times\{0\}}-\lambda^{1-2 s} \partial_{\lambda} v\left\langle x, \nabla_{x} v\right\rangle d x \\
& \quad-\frac{1}{2} \int_{\partial^{+} \widetilde{B}_{R}^{+}} \lambda^{1-2 s}|\nabla v|^{2}\langle z, \nu\rangle d \mathcal{H}^{n}+\frac{n-2 s}{2} \int_{\widetilde{B}_{R}^{+}} \lambda^{1-2 s}|\nabla v|^{2} d x d \lambda=0,
\end{aligned}
$$

where $\nu$ denotes the outer unit normal vector to $\partial^{+} \widetilde{B}_{R}^{+}$.

Now, we use that $z=R \nu$ on $\partial^{+} \widetilde{B}_{R}^{+}$and $-d_{s} \lambda^{1-2 s} \partial_{\lambda} v=f(v)$ on $B_{R}$, to get

$$
\begin{aligned}
& R \int_{\partial^{+} \widetilde{B}_{R}^{+}} \lambda^{1-2 s}\left(\frac{\partial v}{\partial \nu}\right)^{2} d \mathcal{H}^{n}+\int_{B_{R} \times\{0\}} d_{s}^{-1} f(v)\left\langle x, \nabla_{x} v\right\rangle d x \\
& \quad-\frac{R}{2} \int_{\partial^{+} \widetilde{B}_{R}^{+}} \lambda^{1-2 s}|\nabla v|^{2} d \mathcal{H}^{n}+\frac{n-2 s}{2} \int_{\widetilde{B}_{R}^{+}} \lambda^{1-2 s}|\nabla v|^{2} d z=0 .
\end{aligned}
$$

We conclude the proof, observing that, on $\{\lambda=0\}$,

$$
\begin{array}{rl}
\int_{B_{R}} & f(v)\left\langle x, \nabla_{x} v\right\rangle d x=-\int_{B_{R}}\left\langle x, \nabla_{x} G(v)\right\rangle d x=\int_{B_{R}}(-\operatorname{div}(x G(v))+n G(v)) d x \\
=n \int_{B_{R}} G(v) d x-R \int_{\partial B_{R}} G(v) d \mathcal{H}^{n-1} .
\end{array}
$$

We can now prove the monotonicity formula.

Proposition 3.2. Let $s \in(0,1), f$ be any $C^{1, \gamma}$ nonlinearity with $\gamma>\max \{0,1-2 s\}$, and suppose that $v$ is a bounded solution of problem (1.2), and that $G(t) \geq 0$ for every $t \in \mathbb{R}$. 
Then, the function

$$
\phi(R)=\frac{1}{R^{n-2 s}}\left\{\frac{d_{s}}{2} \int_{\widetilde{B}_{R}^{+}} \lambda^{1-2 s}|\nabla v|^{2} d x d \lambda+\int_{B_{R} \times\{0\}} G(v) d x\right\}
$$

is a nondecreasing function of $R>0$.

Proof. We have that

$$
\begin{gathered}
\phi^{\prime}(R)=-\frac{(n-2 s) d_{s}}{2 R^{n-2 s+1}} \int_{\widetilde{B}_{R}^{+}} \lambda^{1-2 s}|\nabla v|^{2} d x d \lambda+\frac{d_{s}}{2 R^{n-2 s}} \int_{\partial^{+} \widetilde{B}_{R}^{+}} \lambda^{1-2 s}|\nabla v|^{2} d \mathcal{H}^{n} \\
-\frac{n-2 s}{R^{n-2 s+1}} \int_{B_{R} \times\{0\}} G(v) d x+\frac{1}{R^{n-2 s}} \int_{\partial B_{R} \times\{0\}} G(v) d \mathcal{H}^{n-1} .
\end{gathered}
$$

Using the Pohozaev identity of Lemma 3.1, we get

$$
\phi^{\prime}(R)=\frac{d_{s}}{R^{n-2 s}} \int_{\partial^{+} \widetilde{B}_{R}^{+}} \lambda^{1-2 s}\left(\frac{\partial v}{\partial \nu}\right)^{2} d \mathcal{H}^{n}+\frac{2 s}{R^{n-2 s+1}} \int_{B_{R} \times\{0\}} G(v) d x \geq 0 .
$$

Remark 3.3. Since

$$
\mathcal{E}_{\widetilde{B}_{R}^{+}}(v) \leq \mathcal{E}_{C_{R}}(v),
$$

Proposition 3.2 gives that, for every bounded solution $v$ of problem (1.2) which is not identically zero, the following lower bound holds:

$$
d_{s} \int_{C_{R}} \frac{1}{2} \lambda^{1-2 s}|\nabla v|^{2} d x d \lambda+\int_{B_{R}}\left\{G(u)-c_{u}\right\} d x \geq c_{1} R^{n-2 s},
$$

for some constant $c_{1}>0$ depending on $v$. Note that Theorem 1.2 establishes $\mathcal{E}_{C_{R}}(v) \leq$ $C R^{n-2 s}$ for every $s \in(0,1 / 2)$. Thus, this bound is sharp as a consequence of (3.1).

\section{4. $H^{s}$ estimate}

In this section we recall some definitions and properties of the spaces $H^{s}\left(\mathbb{R}^{n}\right)$ and $H^{s}(\partial \Omega)$, where $\Omega$ is a bounded subset of $\mathbb{R}^{n+1}$ with Lipschitz boundary $\partial \Omega$ (see [18]).

$H^{s}\left(\mathbb{R}^{n}\right)$ is the space of functions $u \in L^{2}\left(\mathbb{R}^{n}\right)$ such that

$$
\int_{\mathbb{R}^{n}} \int_{\mathbb{R}^{n}} \frac{|u(x)-u(\bar{x})|^{2}}{|x-\bar{x}|^{n+2 s}} d x d \bar{x}<+\infty
$$

equipped with the norm

$$
\|u\|_{H^{s}\left(\mathbb{R}^{n}\right)}=\left(\|u\|_{L^{2}\left(\mathbb{R}^{n}\right)}^{2}+\int_{\mathbb{R}^{n}} \int_{\mathbb{R}^{n}} \frac{|u(x)-u(\bar{x})|^{2}}{|x-\bar{x}|^{n+2 s}} d x d \bar{x}\right)^{\frac{1}{2}} .
$$


As in section 3 of [4], using a family of charts and a partition of unity, we can define the space $H^{s}(\partial \Omega)$, where $\Omega$ is a bounded subset of $\mathbb{R}^{n+1}$ with Lipschitz boundary. We use the same notations of [4].

Consider an atlas $\left\{\left(O_{j}, \varphi_{j}\right), j=1, \ldots, m\right\}$ where $\left\{O_{j}\right\}$ is a family of open bounded sets in $\mathbb{R}^{n+1}$ such that $\left\{O_{j} \cap \partial \Omega ; j=1, \ldots, m\right\}$ cover $\partial \Omega$. The functions $\varphi_{j}$ are corresponding bilipschitz diffeomorphisms such that

- $\varphi_{j}: O_{j} \rightarrow U:=\left\{(y, \mu) \in \mathbb{R}^{n+1}:|y|<1,-1<\mu<1\right\}$,

- $\varphi_{j}: O_{j} \cap \Omega \rightarrow U^{+}:=\left\{(y, \mu) \in \mathbb{R}^{n+1}:|y|<1,0<\mu<1\right\}$, and

- $\varphi_{j}: O_{j} \cap \partial \Omega \rightarrow\left\{(y, \mu) \in \mathbb{R}^{n+1}:|y|<1, \mu=0\right\}$.

Let $\left\{\alpha_{j}\right\}$ be a partition of unity on $\partial \Omega$ such that $0 \leq \alpha_{j} \in C_{c}^{\infty}\left(O_{j}\right), \sum_{j=1}^{m} \alpha_{j}=1$ on $\partial \Omega$. If $u$ is a function on $\partial \Omega$, decompose $u=\sum_{j=1}^{m} u \alpha_{j}$ and define the function

$$
\left(u \alpha_{j}\right) \circ \varphi_{j}^{-1}(y, 0):=\left(u \alpha_{j}\right)\left(\varphi_{j}^{-1}(y, 0)\right), \quad \text { for every }(y, 0) \in U \cap\{\mu=0\} .
$$

Since $\alpha_{j}$ has compact support in $O_{j}$, the function $\left(u \alpha_{j}\right) \circ \varphi_{j}^{-1}(\cdot, 0)$ has compact support in $U \cap\{\mu=0\}$ and therefore we may consider $\left(\left(u \alpha_{j}\right) \circ \varphi_{j}^{-1}\right)(\cdot, 0)$ to be defined in $\mathbb{R}^{n}$ extending it by zero out of $U \cap\{\mu=0\}$. Now we define

$$
H^{s}(\partial \Omega):=\left\{u \mid\left(u \alpha_{j}\right) \circ \varphi_{j}^{-1}(\cdot, 0) \in H^{s}\left(\mathbb{R}^{n}\right), j=1, \ldots, m\right\}
$$

equipped with the norm

$$
\left(\sum_{j=1}^{m}\left\|\left(u \alpha_{j}\right) \circ \varphi_{j}^{-1}(\cdot, 0)\right\|_{H^{s}\left(\mathbb{R}^{n}\right)}^{2}\right)^{\frac{1}{2}} .
$$

Independently of the choice of the system of local maps $\left\{O_{j}, \varphi_{j}\right\}$ and of the partition of unity $\left\{\alpha_{j}\right\}$, these norms are all equivalent to

$$
\|u\|_{H^{s}(\partial \Omega)}:=\left(\|u\|_{L^{2}(\partial \Omega)}^{2}+\int_{\partial \Omega} \int_{\partial \Omega} \frac{|u(z)-u(\bar{z})|^{2}}{|z-\bar{z}|^{n+2 s}} d \mathcal{H}^{n}(z) d \mathcal{H}^{n}(\bar{z})\right)^{\frac{1}{2}} .
$$

We can give now the proof of Theorem 1.7 .

Proof of Theorem 1.7. Let $s \in(0,1)$.

Case 1: $\Omega=\mathbb{R}_{+}^{n+1}$. We first consider the case of a half space $\Omega=\mathbb{R}_{+}^{n+1}$ and $M=\left\{\left(x^{\prime}, x_{n}\right) \in \mathbb{R}^{n}: x_{n}<0\right\}$. Let $\zeta$ be a bounded function belonging to $C\left(\mathbb{R}^{n}\right)$. Following the first part of the proof of Proposition 3.1 in [4], we consider a $C^{\infty}$ function $K(x)$, defined on $\mathbb{R}^{n}$ with compact support in $B_{1}$ and such that $\int_{\mathbb{R}^{n}} K(x) d x=1$. Define $\widetilde{K}(x, \lambda)$ on $\mathbb{R}_{+}^{n+1}$ in the following way:

$$
\widetilde{K}(x, \lambda):=\frac{1}{\lambda^{n}} K\left(\frac{x}{\lambda}\right)
$$


and finally define the extension $\widetilde{\zeta}$ as

$$
\widetilde{\zeta}(x, \lambda)=\int_{\mathbb{R}^{n}} \widetilde{K}(x-\bar{x}, \lambda) \zeta(\bar{x}) d \bar{x}
$$

Note that, since $\int_{\mathbb{R}^{n}} \widetilde{K}(x, \lambda) d x=1$, we have

$$
\|\widetilde{\zeta}(\cdot, \lambda)\|_{L^{2}\left(\mathbb{R}^{n}\right)} \leq\|\zeta\|_{L^{2}\left(\mathbb{R}^{n}\right)} \text { for every } \lambda \geq 0
$$

and thus

$$
\int_{0}^{1} d \lambda \lambda^{1-2 s} \int_{\mathbb{R}^{n}} d x|\widetilde{\zeta}(x, \lambda)|^{2} \leq \frac{1}{2(1-s)}\|\zeta\|_{L^{2}\left(\mathbb{R}^{n}\right)}^{2}
$$

In [4] (see the proof of Proposition 3.1), a simple calculation led to the following estimate for the gradient of $\widetilde{\zeta}$ :

$$
|\nabla \widetilde{\zeta}(x, \lambda)|^{2} \leq C \int_{\{|x-\bar{x}|<\lambda\}} \frac{|\zeta(x)-\zeta(\bar{x})|^{2}}{\lambda^{n+2}} d \bar{x},
$$

where $\nabla$ denotes the gradient with respect to $x$ and to $\lambda$, and $C$ depends only on $n$.

Since $M=\left\{\left(x^{\prime}, x_{n}\right) \in \mathbb{R}^{n}: x_{n}<0\right\}$, we have $d_{M}(x, \lambda)=\left(\left(x_{n}\right)_{+}^{2}+\lambda^{2}\right)^{1 / 2}$, where as usually $\left(x_{n}\right)_{+}=\max \left\{x_{n}, 0\right\}$.

Consider now, separately, the two cases $0<s \leq 1 / 2$ and $1 / 2<s<1$.

If $0<s \leq 1 / 2$ then $a=1-2 s \in[0,1)$ and we have that $d_{M}^{a}(x, \lambda) \leq\left(x_{n}\right)_{+}^{a}+\lambda^{a}$. In the following computations $C$ will denote different positive constants which may depend on $s$. Using (4.4), we have

$$
\begin{aligned}
& \int_{\mathbb{R}_{+}^{n+1}} d_{M}^{a}(x, \lambda)|\nabla \widetilde{\zeta}(x, \lambda)|^{2} d x d \lambda \leq \int_{\mathbb{R}_{+}^{n+1}}\left(\left(x_{n}\right)_{+}^{a}+\lambda^{a}\right)|\nabla \widetilde{\zeta}(x, \lambda)|^{2} d x d \lambda \\
& \leq C \int_{0}^{+\infty} d \lambda \iint_{\{|x-\bar{x}|<\lambda\}} d x d \bar{x} \frac{\left(x_{n}\right)_{+}^{a}+\lambda^{a}}{\lambda^{n+2}}|\zeta(x)-\zeta(\bar{x})|^{2} \\
& \leq C \int_{0}^{+\infty} d \lambda \iint_{\{|x-\bar{x}|<\lambda\}} d x d \bar{x} \frac{1}{\lambda^{n+2-a}}|\zeta(x)-\zeta(\bar{x})|^{2} \\
&+C \int_{0}^{+\infty} d \lambda \iint_{\{|x-\bar{x}|<\lambda\}} d x d \bar{x} \frac{\left(x_{n}\right)_{+}^{a}}{\lambda^{n+2}}|\zeta(x)-\zeta(\bar{x})|^{2} \\
& \leq C \int_{\mathbb{R}^{n}} \int_{\mathbb{R}^{n}} d x d \bar{x}|\zeta(x)-\zeta(\bar{x})|^{2}\left[\lambda^{-n-1+a}\right]_{+\infty}^{|x-\bar{x}|} \\
&+C \int_{\mathbb{R}^{n}} \int_{\mathbb{R}^{n}} d x d \bar{x}\left(x_{n}\right)_{+}^{a}|\zeta(x)-\zeta(\bar{x})|^{2}\left[\lambda^{-n-1}\right]_{+\infty}^{|x-\bar{x}|} \\
& \leq C \int_{\mathbb{R}^{n}} \int_{\mathbb{R}^{n}} \frac{|\zeta(x)-\zeta(\bar{x})|^{2}}{|x-\bar{x}|^{n+2 s} d x d \bar{x}} \\
&+C \int_{\mathbb{R}^{n}} \int_{\mathbb{R}^{n}}\left(x_{n}\right)_{+}^{1-2 s} \frac{|\zeta(x)-\zeta(\bar{x})|^{2}}{|x-\bar{x}|^{n+1}} d x d \bar{x} .
\end{aligned}
$$


Next, we observe that, in this bound, the last integral can be computed only on the set $\left\{(x, \bar{x}) \in \mathbb{R}^{n} \times \mathbb{R}^{n}:|x-\bar{x}|<\left(x_{n}\right)_{+} / 2\right\}$, which is contained in $\left(\mathbb{R}^{n} \backslash M\right) \times\left(\mathbb{R}^{n} \backslash M\right)$. Indeed

$$
\begin{aligned}
& \iint_{\left\{|x-\bar{x}| \geq \frac{\left(x_{n}\right)_{+}}{2}\right\}}\left(x_{n}\right)_{+}^{1-2 s} \frac{|\zeta(x)-\zeta(\bar{x})|^{2}}{|x-\bar{x}|^{n+1}} d x d \bar{x} \\
& \quad \leq 2^{1-2 s} \iint_{\left\{|x-\bar{x}| \geq \frac{\left(x_{n}\right)_{+}}{2}\right\}} \frac{|\zeta(x)-\zeta(\bar{x})|^{2}}{|x-\bar{x}|^{n+2 s}} d x d \bar{x}
\end{aligned}
$$

which can be absorbed in the first integral of the above bound.

Thus, if $0<s \leq 1 / 2$, we have

$$
\begin{aligned}
& \int_{\mathbb{R}_{+}^{n+1}} d_{M}(x, \lambda)^{1-2 s}|\nabla \widetilde{\zeta}(x, \lambda)|^{2} d x d \lambda \\
& \leq C \int_{\mathbb{R}^{n}} \int_{\mathbb{R}^{n}} \frac{|\zeta(x)-\zeta(\bar{x})|^{2}}{|x-\bar{x}|^{n+2 s}} d x d \bar{x}+C \int_{\mathbb{R}^{n} \backslash M} \int_{\mathbb{R}^{n} \backslash M} d_{M}(x)^{1-2 s} \frac{|\zeta(x)-\zeta(\bar{x})|^{2}}{|x-\bar{x}|^{n+1}} d x d \bar{x}
\end{aligned}
$$

If $1 / 2<s<1$, set

$$
b=-a=2 s-1>0 .
$$

In this case we use that $d_{M}(x, \lambda) \geq \max \left\{\left(x_{n}\right)_{+}, \lambda\right\}$, which leads to

$$
d_{M}^{a}(x, \lambda)=1 / d_{M}^{b}(x, \lambda) \leq 1 /\left(\max \left\{\left(x_{n}\right)_{+}, \lambda\right\}\right)^{b} .
$$

In what follows we will use $d_{M}^{a}(x, \lambda) \leq 1 / \lambda^{b}$ if $\left(x_{n}\right)_{+}=0$ and $d_{M}^{a}(x, \lambda) \leq 1 /\left(x_{n}\right)_{+}^{b}$ if $\left(x_{n}\right)_{+}>0$. We have

$$
\begin{aligned}
& \int_{\mathbb{R}_{+}^{n+1}} d_{M}^{a}(x, \lambda)|\nabla \widetilde{\zeta}(x, \lambda)|^{2} d x d \lambda \leq \\
& \leq C \int_{0}^{+\infty} d \lambda \int_{\left\{\left(x_{n}\right)_{+}=0\right\}} \int_{\{|x-\bar{x}|<\lambda\}} d x d \bar{x} \frac{|\zeta(x)-\zeta(\bar{x})|^{2}}{\lambda^{n+2+b}} \\
&+C \int_{0}^{+\infty} d \lambda \int_{\left\{\left(x_{n}\right)_{+}>0\right\}} \int_{\{|x-\bar{x}|<\lambda\}} d x d \bar{x} \frac{|\zeta(x)-\zeta(\bar{x})|^{2}}{\left(x_{n}\right)_{+}^{b} \lambda^{n+2}} \\
& \leq C \int_{\left\{\left(x_{n}\right)_{+}=0\right\}} d x \int_{\mathbb{R}^{n}} d \bar{x} \frac{|\zeta(x)-\zeta(\bar{x})|^{2}}{|x-\bar{x}| n+2 s} \\
&+C \int_{\left\{\left(x_{n}\right)_{+}>0\right\}} d x \int_{\mathbb{R}^{n}} d \bar{x} \frac{1}{\left(x_{n}\right)_{+}^{b}} \frac{|\zeta(x)-\zeta(\bar{x})|^{2}}{|x-\bar{x}|^{n+1}} \\
& \leq C \int_{\left\{\left(x_{n}\right)_{+}=0\right\}} d x \int_{\left\{\left(\bar{x}_{n}\right)_{+}=0\right\}} d \bar{x} \frac{|\zeta(x)-\zeta(\bar{x})|^{2}}{|x-\bar{x}|^{n+2 s}} \\
&+C \int_{\left\{\left(x_{n}\right)_{+}>0\right\}} d x \int_{\mathbb{R}^{n}} d \bar{x} \frac{1}{\left(x_{n}\right)_{+}^{b}} \frac{|\zeta(x)-\zeta(\bar{x})|^{2}}{|x-\bar{x}|^{n+1}} .
\end{aligned}
$$


Observe that the integral in (4.5) is computed only on the set $\left\{(x, \bar{x}) \in \mathbb{R}^{n} \times\right.$ $\left.\mathbb{R}^{n} \mid\left(x_{n}\right)_{+}=0,\left(\bar{x}_{n}\right)_{+}=0\right\}$. Indeed the set $L:=M \times\left(\mathbb{R}^{n} \backslash M\right)=\{(x, \bar{x}) \in$ $\left.\mathbb{R}^{n} \times \mathbb{R}^{n} \mid\left(x_{n}\right)_{+}=0,\left(\bar{x}_{n}\right)_{+}>0\right\} \subseteq\left\{(x, \bar{x}) \in \mathbb{R}^{n} \times \mathbb{R}^{n}\left|\left(\bar{x}_{n}\right)_{+} \leq\right| x-\bar{x} \mid\right\}$. Then if $(x, \bar{x}) \in L$

$$
\frac{1}{|x-\bar{x}|^{n+1+b}} \leq \frac{1}{\left(\bar{x}_{n}\right)_{+}^{b}} \cdot \frac{1}{|x-\bar{x}|^{n+1}}
$$

and hence we have that

$\int_{\left\{\left(x_{n}\right)_{+}=0\right\}} d x \int_{\left\{\left(\bar{x}_{n}\right)_{+}>0\right\}} d \bar{x} \frac{|\zeta(x)-\zeta(\bar{x})|^{2}}{|x-\bar{x}|^{n+2 s}} \leq C \int_{\left\{\left(\bar{x}_{n}\right)_{+}>0\right\}} d \bar{x} \int_{\mathbb{R}^{n}} d x \frac{1}{\left(\bar{x}_{n}\right)_{+}^{b}} \frac{|\zeta(x)-\zeta(\bar{x})|^{2}}{|x-\bar{x}|^{n+1}}$, which is equal to the integral in (4.6). This concludes the proof in the case of the half space.

Case 2: Let $\Omega \subset \mathbb{R}^{n+1}$ be a bounded domain with Lipschitz boundary $A=\partial \Omega$, and let $w \in C(\partial \Omega)$.

Let $\Gamma$ be the boundary (relative to $A=\partial \Omega$ ) of $M$ and let $\widetilde{B}_{r_{i}}=\widetilde{B}_{r_{i}}\left(p_{i}\right) \subset \mathbb{R}^{n+1}$ be the ball centered at $p_{i} \in \partial \Omega$ and of radius $r_{i}$. We set $A_{r_{i}}:=\widetilde{B}_{r_{i}} \cap \partial \Omega$. Let $Q_{1}$ denote the unite cube in $\mathbb{R}^{n}$.

Since $\partial \Omega$ is compact, we can consider a finite open covering of $\partial \Omega$,

$$
\bigcup_{i=1}^{m} A_{r_{i}}:=\bigcup_{i=1}^{m}\left(\widetilde{B}_{r_{i}} \cap \partial \Omega\right)
$$

such that for every $i$ there exists a bilipschitz function $\varphi_{i}: \widetilde{B}_{r_{i}} \rightarrow Q_{1} \times(-1,1)$ which satisfies

$$
\varphi_{i}\left(\widetilde{B}_{r_{i}} \cap \Omega\right)=Q_{1} \times(0,1) \text { and } \varphi_{i}\left(A_{r_{i}}\right)=Q_{1} \times\{0\} .
$$

Moreover we may require that

- if $\Gamma_{i}=A_{r_{i}} \cap \Gamma \neq \emptyset$, then

$$
\begin{aligned}
\varphi_{i}\left(\Gamma_{i}\right) & =\left\{x \in Q_{1}: x_{n}=0\right\} ; \\
\varphi_{i}\left(M \cap A_{r_{i}}\right) & =\left\{x \in Q_{1}: x_{n}<0\right\}=Q_{1}^{-} ;
\end{aligned}
$$

- if $\bar{A}_{r_{i}} \cap M=\emptyset$, then

$$
r_{i}=\frac{1}{3} d_{M}\left(p_{i}\right)
$$

where $p_{i}$ and $r_{i}$ are respectively the center and the radius of the ball $\widetilde{B}_{r_{i}}$.

Observe that the number $m$ of sets $A_{r_{i}}$ which cover $\partial \Omega$, and the Lipschitz constants of $\varphi_{i}$, depend only on $\partial \Omega$ and $\Gamma$.

We consider a partition of unity $\left\{\alpha_{i}\right\}_{i=1, \ldots, m}$ relative to the covering $\left\{\widetilde{B}_{r_{i}}\right\}_{i=1, \ldots, m}$, where $\alpha_{i} \in C_{c}^{\infty}\left(\widetilde{B}_{r_{i}}\right)$ and $\sum_{i=1}^{m} \alpha_{i}=1$ on $\partial \Omega$. 
If $w$ is a function defined on $\partial \Omega$, we write

$$
w=\sum_{i=1}^{m} w \alpha_{i}=\sum_{i=1}^{m} w_{i}
$$

Using the bilipschitz map $\varphi_{i}$, we define

$$
\zeta_{i}(y):=w_{i}\left(\varphi_{i}^{-1}(y, 0)\right) \quad \text { for every } y \in Q_{1} .
$$

Then $\zeta_{i}$ has compact support in $Q_{1}$ and we extend it by 0 outside $Q_{1}$ in all $\mathbb{R}^{n}$.

Next, we consider $\widetilde{\zeta}_{i}$, the extension of $\zeta_{i}$ in $\mathbb{R}_{+}^{n+1}$ defined by the convolution in (4.1). Since $\alpha_{i} \in C_{c}^{\infty}\left(\widetilde{B}_{r_{i}}\right)$, there exists a function $\beta_{i} \in C_{c}^{\infty}\left(\widetilde{B}_{r_{i}}\right)$ such that $\beta_{i} \equiv 1$ in the support of $\alpha_{i}$. Thus $\widetilde{w}_{i}:=\beta_{i}\left(\widetilde{\zeta}_{i} \circ \varphi_{i}\right)$, extended by zero outside of $\widetilde{B}_{r_{i}}$, is well defined as a function in $\bar{\Omega}$ and agrees with $w \alpha_{i}=\beta_{i} w \alpha_{i}$ on $\partial \Omega$. We now define

$$
\widetilde{w}=\sum_{i=1}^{m} \widetilde{w}_{i}=\sum_{i=1}^{m} \beta_{i}\left(\widetilde{\zeta}_{i} \circ \varphi_{i}\right) \text { in } \bar{\Omega}
$$

which agrees with $w$ on $\partial \Omega$.

Observe that, since $\varphi_{i}$ is a bilipschitz map and $\alpha_{i}, \beta_{i} \in C_{c}^{\infty}\left(\widetilde{B}_{r_{i}}\right)$ for every $i=$ $1, \ldots, m$, we have

$$
\left|\nabla \widetilde{w}_{i}\right| \leq C\left\{\left|\nabla \beta_{i}\right|\left|\widetilde{\zeta}_{i} \circ \varphi_{i}\right|+\left|\beta_{i}\right|\left|\left(\nabla \widetilde{\zeta}_{i}\right) \circ \varphi_{i}\right|\right\}
$$

and thus

$$
\begin{aligned}
\int_{\widetilde{B}_{r_{i}} \cap \Omega} d_{M}^{1-2 s}(z)\left|\nabla \widetilde{w}_{i}\right|^{2} d z \leq C & \int_{\widetilde{B}_{r_{i}} \cap \Omega} d_{M}^{1-2 s}(z)\left|\widetilde{\zeta}_{i} \circ \varphi_{i}\right|^{2} d z \\
& +C \int_{\widetilde{B}_{r_{i}} \cap \Omega} d_{M}^{1-2 s}(z)\left|\left(\nabla \widetilde{\zeta}_{i}\right) \circ \varphi_{i}\right|^{2} d z .
\end{aligned}
$$

Observe that when $0<s \leq 1 / 2$, we have that $d_{M}^{1-2 s}$ is uniformly bounded in $\Omega$, and thus using (4.2) we get

$$
\begin{aligned}
& \int_{\widetilde{B}_{r_{i}} \cap \Omega} d_{M}^{1-2 s}(z)\left|\widetilde{\zeta}_{i} \circ \varphi_{i}\right|^{2} d z \leq C \int_{\widetilde{B}_{r_{i}} \cap \Omega}\left|\widetilde{\zeta}_{i} \circ \varphi_{i}\right|^{2} d z \\
& \quad \leq C\|\zeta\|_{L^{2}\left(\mathbb{R}^{n}\right)}^{2} \leq C|| w \|_{L^{2}(\partial \Omega) .}^{2} .
\end{aligned}
$$

On the other hand, when $1 / 2<s<1$, we use (4.3), to obtain

$$
\begin{aligned}
& \int_{\widetilde{B}_{r_{i}} \cap \Omega} d_{M}^{1-2 s}(z)\left|\widetilde{\zeta}_{i} \circ \varphi_{i}\right|^{2} d z \leq C \int_{0}^{1} \int_{Q_{1}} \lambda^{1-2 s}\left|\widetilde{\zeta}_{i}(x, \lambda)\right|^{2} d x d \lambda \\
& \quad \leq C\|\zeta\|_{L^{2}\left(\mathbb{R}^{n}\right)}^{2} \leq C\|w\|_{L^{2}(\partial \Omega) .}^{2} .
\end{aligned}
$$


Thus

$$
\int_{\widetilde{B}_{r_{i}} \cap \Omega} d_{M}^{1-2 s}(z)\left|\nabla \widetilde{w}_{i}\right|^{2} d z \leq\left. C|| w\right|_{L^{2}(\partial \Omega)} ^{2}+C \int_{\widetilde{B}_{r_{i}} \cap \Omega} d_{M}^{1-2 s}(z)\left|\left(\nabla \widetilde{\zeta}_{i}\right) \circ \varphi_{i}\right|^{2} d z .
$$

Using this bound we can prove the following

Claim: (1.13) holds with $\widetilde{w}$ and $w$ replaced by $\widetilde{w}_{i}$ and $w_{i}$, which have compact support in $\widetilde{B}_{r_{i}} \cap \bar{\Omega}$ and $A_{r_{i}}$ respectively.

It is enough to prove the claim to conclude the proof. Indeed, note first that

$$
\int_{\Omega} d_{M}(z)^{1-2 s}|\nabla \widetilde{w}|^{2} d z \leq C \sum_{i=1}^{m} \int_{\widetilde{B}_{r_{i}} \cap \Omega} d_{M}(z)^{1-2 s}\left|\nabla \widetilde{w}_{i}\right|^{2} d z .
$$

Moreover, for every $i=1, \ldots, m$,

$$
\begin{aligned}
& \iint_{B_{\mathrm{frac}}} \frac{\left|w_{i}(z)-w_{i}(\bar{z})\right|^{2}}{|z-\bar{z}|^{n+2 s}} d \mathcal{H}^{n}(z) d \mathcal{H}^{n}(\bar{z}) \\
& \leq C|| w \|_{L^{2}(\partial \Omega)}^{2}+C \iint_{B_{\mathrm{frac}}} \frac{|w(z)-w(\bar{z})|^{2}}{|z-\bar{z}|^{n+2 s}} d \mathcal{H}^{n}(z) d \mathcal{H}^{n}(\bar{z}) .
\end{aligned}
$$

Indeed,

$$
\begin{aligned}
\int & \int_{B_{\mathrm{frac}}} \frac{\left|\left(w \alpha_{i}\right)(z)-\left(w \alpha_{i}\right)(\bar{z})\right|^{2}}{|z-\bar{z}|^{n+2 s}} d \mathcal{H}^{n}(z) d \mathcal{H}^{n}(\bar{z}) \\
= & \iint_{B_{\mathrm{frac}}} \frac{\left|\left(w \alpha_{i}\right)(z)-w(\bar{z}) \alpha_{i}(z)+w(\bar{z}) \alpha_{i}(z)-\left(w \alpha_{i}\right)(\bar{z})\right|^{2}}{|z-\bar{z}|^{n+2 s}} d \mathcal{H}^{n}(z) d \mathcal{H}^{n}(\bar{z}) \\
\leq & 2 \iint_{B_{\mathrm{frac}}} \frac{\left|\alpha_{i}(z)-\alpha_{i}(\bar{z})\right|^{2}|w(\bar{z})|^{2}}{|z-\bar{z}|^{n+2 s}} d \mathcal{H}^{n}(z) d \mathcal{H}^{n}(\bar{z}) \\
& +2 \iint_{B_{\mathrm{frac}}} \frac{|w(z)-w(\bar{z})|^{2}\left|\alpha_{i}(z)\right|^{2}}{|z-\bar{z}|^{n+2 s}} d \mathcal{H}^{n}(z) d \mathcal{H}^{n}(\bar{z}) \\
\leq & C\|w\|_{L^{2}(\partial \Omega)}^{2}+C \iint_{B_{\mathrm{frac}}} \frac{|w(z)-w(\bar{z})|^{2}}{|z-\bar{z}|^{n+2 s}} d \mathcal{H}^{n}(z) d \mathcal{H}^{n}(\bar{z})
\end{aligned}
$$

where $C$ denotes different positive constants depending on $\Omega$ and $s$. To get the bound $C\|w\|_{L^{2}(\partial \Omega)}^{2}$ for the first term, we have used spherical coordinates centered at $\bar{z}$ and that $\alpha_{i}$ is Lipschitz.

Arguing in the same way, we deduce

$$
\begin{aligned}
& \iint_{B_{\text {weig }}} d_{M}(z)^{1-2 s} \frac{\left|w_{i}(z)-w_{i}(\bar{z})\right|^{2}}{|z-\bar{z}|^{n+1}} d \mathcal{H}^{n}(z) d \mathcal{H}^{n}(\bar{z}) \\
& \quad \leq C\|w\|_{L^{2}(\partial \Omega)}^{2}+C \iint_{B_{\text {weig }}} d_{M}(z)^{1-2 s} \frac{|w(z)-w(\bar{z})|^{2}}{|z-\bar{z}|^{n+1}} d \mathcal{H}^{n}(z) d \mathcal{H}^{n}(\bar{z}) .
\end{aligned}
$$


Indeed, using spherical coordinates centered at $\bar{z}$, that $\alpha_{i}$ is Lipschitz, and the definition (1.12) of $B_{\text {weig }}$, we deduce (after flattening the boundary)

$$
\begin{aligned}
& \iint_{B_{\text {weig }}} d_{M}(z)^{1-2 s} \frac{\left|\left(w \alpha_{i}\right)(z)-\left(w \alpha_{i}\right)(\bar{z})\right|^{2}}{|z-\bar{z}|^{n+1}} d \mathcal{H}^{n}(z) d \mathcal{H}^{n}(\bar{z}) \\
& \leq 2 \iint_{B_{\text {weig }}} d_{M}(z)^{1-2 s} \frac{\left|\alpha_{i}(z)-\alpha_{i}(\bar{z})\right|^{2}|w(\bar{z})|^{2}}{|z-\bar{z}|^{n+1}} d \mathcal{H}^{n}(z) d \mathcal{H}^{n}(\bar{z}) \\
& \quad+2 \iint_{B_{\text {weig }}} d_{M}(z)^{1-2 s} \frac{|w(z)-w(\bar{z})|^{2}\left|\alpha_{i}(z)\right|^{2}}{|z-\bar{z}|^{n+1}} d \mathcal{H}^{n}(z) d \mathcal{H}^{n}(\bar{z}) \\
& \leq C|| w \|_{L^{2}(\partial \Omega)}^{2}+C \iint_{B_{\text {weig }}} d_{M}(z)^{1-2 s} \frac{|w(z)-w(\bar{z})|^{2}}{|z-\bar{z}|^{n+1}} d \mathcal{H}^{n}(z) d \mathcal{H}^{n}(\bar{z}) .
\end{aligned}
$$

Next, we prove the claim.

Observe that we have three different cases, depending on the relative positions between the sets $A_{r_{i}}$ and $M$.

Case a). First, consider the case $\Gamma_{i}=A_{r_{i}} \cap \Gamma \neq \emptyset$. By (4.10), we have that

$$
\begin{aligned}
& \int_{\widetilde{B}_{r_{i}} \cap \Omega} d_{M}(z)^{1-2 s}\left|\nabla \widetilde{w}_{i}\right|^{2} d z \\
& \quad \leq C|| w \|_{L^{2}(\partial \Omega)}^{2}+C \int_{\mathbb{R}_{+}^{n+1}}\left(\left(x_{n}\right)_{+}+\lambda\right)^{1-2 s}\left|\nabla \widetilde{\zeta}_{i}\right|^{2} d x d \lambda .
\end{aligned}
$$

Then, using the result in case 1 , applied to $\widetilde{\zeta}_{i}$, we get

$$
\begin{aligned}
\int_{\widetilde{B}_{r_{i}} \cap \Omega} & d_{M}(z)^{1-2 s}\left|\nabla \widetilde{w}_{i}\right|^{2} d z \\
\leq & C \||w|_{L^{2}(\partial \Omega)}^{2}+C \int_{\mathbb{R}_{+}^{n+1}}\left(\left(x_{n}\right)_{+}+\lambda\right)^{1-2 s}\left|\nabla \widetilde{\zeta}_{i}\right|^{2} d x d \lambda \\
\leq & C\|w\|_{L^{2}(\partial \Omega)}^{2}+C \iint_{B_{\text {frac }}} \frac{\left|\zeta_{i}(x)-\zeta_{i}(\bar{x})\right|^{2}}{|x-\bar{x}|^{n+2 s}} d x d \bar{x} \\
& +C \iint_{B_{\text {weig }}}\left(x_{n}\right)_{+}^{1-2 s} \frac{\mid \zeta_{i}(x)-\zeta_{i}\left(\left.\bar{x}\right|^{2}\right.}{|x-\bar{x}|^{n+1}} d x d \bar{x}
\end{aligned}
$$

where $B_{\text {frac }}$ and $B_{\text {weig }}$ are defined as in (1.11) and (1.12) with $A=\mathbb{R}^{n}$ and $M=$ $\left\{\left(x^{\prime}, x_{n}\right) \in \mathbb{R}^{n}: x_{n}<0\right\}$. 
Using the bilipschitz map $\varphi_{i}^{-1}$, we have

$$
\begin{aligned}
\int_{\widetilde{B}_{r_{i}} \cap \Omega} & d_{M}(z)^{1-2 s}\left|\nabla \widetilde{w}_{i}\right|^{2} d z \\
\leq & C|| w \|_{L^{2}(\partial \Omega)}^{2}+C \iint_{B_{\mathrm{frac}}} \frac{\left|w_{i}(z)-w_{i}(\bar{z})\right|^{2}}{|z-\bar{z}|^{n+2 s}} d \mathcal{H}^{n}(z) d \mathcal{H}^{n}(\bar{z}) \\
& +C \iint_{B_{\mathrm{weig}}} d_{M}(z)^{1-2 s} \frac{\left|w_{i}(z)-w_{i}(\bar{z})\right|^{2}}{|z-\bar{z}|^{n+1}} d \mathcal{H}^{n}(z) d \mathcal{H}^{n}(\bar{z}),
\end{aligned}
$$

where now, $B_{\text {frac }}$ and $B_{\text {weig }}$ are defined as in (1.11) and (1.12) with $A=\partial \Omega$.

Case b). Second, consider the case $A_{r_{i}} \subset M$. In this case, the claim follows exactly as in case a), with $\left(x_{n}\right)_{+}=0$ in (4.11).

Case c). Finally, consider the case $A_{r_{i}} \subset \partial \Omega \backslash M$.

We recall that, by construction $A_{r_{i}}=\widetilde{B}_{r_{i}} \cap \partial \Omega$ where $\widetilde{B}_{r_{i}}$ is the ball centered at $p_{i} \in \partial \Omega \backslash M$ and of radius $r_{i}=\frac{1}{3} d_{M}\left(p_{i}\right)$.

Thus, for every $z \in \widetilde{B}_{r_{i}} \cap \Omega$, we have that

$$
\frac{2}{3} d_{M}\left(p_{i}\right) \leq d_{M}(z) \leq \frac{4}{3} d_{M}\left(p_{i}\right) .
$$

Then, for every $i=1, \ldots, m$

$$
\int_{\widetilde{B}_{r_{i}} \cap \Omega} d_{M}(z)^{1-2 s}\left|\nabla \widetilde{w}_{i}\right|^{2} d z \leq C d_{M}\left(p_{i}\right)^{1-2 s} \int_{\widetilde{B}_{r_{i}} \cap \Omega}\left|\nabla \widetilde{w}_{i}\right|^{2} d z .
$$

Observe that the integral on the right-hand side does not contain weights. Moreover, we recall that the extension $\widetilde{w}_{i}$ is defined as for the case $s=1 / 2$. Thus, applying the extension result given in [4] for $s=1 / 2$, we get

$$
\begin{aligned}
\int_{\widetilde{B}_{r_{i}} \cap \Omega} & d_{M}(z)^{1-2 s}\left|\nabla \widetilde{w}_{i}\right|^{2} d z \\
\leq & C\|w\|_{L^{2}(\partial \Omega)}^{2}+C d_{M}\left(p_{i}\right)^{1-2 s} \iint_{B_{\text {weig }}} \frac{\left|w_{i}(z)-w_{i}(\bar{z})\right|^{2}}{|z-\bar{z}|^{n+1}} d \mathcal{H}^{n}(z) d \mathcal{H}^{n}(\bar{z}) \\
\leq & C\|w\|_{L^{2}(\partial \Omega)}^{2}+C \iint_{B_{\text {weig }}} d_{M}(z)^{1-2 s} \frac{\left|w_{i}(z)-w_{i}(\bar{z})\right|^{2}}{|z-\bar{z}|^{n+1}} d \mathcal{H}^{n}(z) d \mathcal{H}^{n}(\bar{z}),
\end{aligned}
$$

where $B_{\text {frac }}$ and $B_{\text {weig }}$ are defined as in (1.11) and (1.12) with $A=\partial \Omega$. This concludes the proof of the claim.

We give now the proof of Theorem 1.9.

Proof of Theorem 1.9. Let $s \in(0,1)$. When $s=1 / 2$ the theorem was proved in our previous work [4], Theorem 1.5. Here we will prove it for $0<s<1 / 2$ and $1 / 2<s<1$. 
The proof differs in each of these two cases, they also differ from the one for $s=1 / 2$ in which there is a $|\log \varepsilon|$ appearing in the final bound.

Step 1. Suppose that $A=Q_{1}=\left\{x \in \mathbb{R}^{n}:|x|<1\right\}$ is the unit cube in $\mathbb{R}^{n}$. Let, as before, $\left(x^{\prime}, x_{n}\right) \in \mathbb{R}^{n}$. As in the proof of Theorem 1.7, we consider $M=Q_{1}^{-}=$ $\left\{x \in Q_{1}: x_{n}<0\right\}$ and $\Gamma=\left\{x \in Q_{1}: x_{n}=0\right\}$. In the following computations $C$ will denote different positive constants which depend on $n, s$, and $c_{s}$.

Case $0<s<1 / 2$. By hypothesis $|w(x)| \leq c_{s}$ and by (1.14) we have

$$
|D w(x)| \leq \begin{cases}\frac{c_{s}}{\varepsilon}\left(\frac{d_{\Gamma}(x)}{\varepsilon}\right)^{2 s-1}=\frac{c_{s}}{\varepsilon}\left(\frac{\left|x_{n}\right|}{\varepsilon}\right)^{2 s-1} & \text { in } \left.A \cap\left|x_{n}\right| \leq \varepsilon\right\} \\ \frac{c_{s}}{d_{\Gamma}(x)}=\frac{c_{s}}{\left|x_{n}\right|} & \text { in } A \cap\left\{\left|x_{n}\right|>\varepsilon\right\} .\end{cases}
$$

Let $Q_{1}^{+}=\left\{x \in Q_{1}: x_{n}>0\right\}$. Following (1.11) and (1.12) we must consider the quantity

$$
\begin{aligned}
I:= & \int_{Q_{1}} \int_{Q_{1}} \frac{|w(x)-w(\bar{x})|^{2}}{|x-\bar{x}|^{n+2 s}} d x d \bar{x} \\
& +\int_{Q_{1}^{+}} \int_{Q_{1}^{+}}\left(x_{n}\right)_{+}^{1-2 s} \frac{|w(x)-w(\bar{x})|^{2}}{|x-\bar{x}|^{n+1}} d x d \bar{x} .
\end{aligned}
$$

Since hypothesis (4.12) is symmetric in $x_{n}$ and $-x_{n}$, we simply bound $I$ by

$$
I \leq \int_{Q_{1}} \int_{Q_{1}} \frac{|w(x)-w(\bar{x})|^{2}}{|x-\bar{x}|^{n+2 s}} d x d \bar{x}+\int_{Q_{1}} \int_{Q_{1}}\left|x_{n}\right|^{1-2 s} \frac{|w(x)-w(\bar{x})|^{2}}{|x-\bar{x}|^{n+1}} d x d \bar{x} .
$$

Observe that, in the set $\left\{|x-\bar{x}|<\left|x_{n}\right| / 2\right\}$, we have

$$
\frac{|w(x)-w(\bar{x})|^{2}}{|x-\bar{x}|^{n+2 s}} \leq 2^{2 s-1}\left|x_{n}\right|^{1-2 s} \frac{|w(x)-w(\bar{x})|^{2}}{|x-\bar{x}|^{n+1}},
$$

while the reverse inequality holds in $\left\{|x-\bar{x}| \geq\left|x_{n}\right| / 2\right\}$. We deduce that

$$
\begin{aligned}
& I \leq C \int_{Q_{1}} \int_{Q_{1} \cap\left\{\bar{x}:|x-\bar{x}|>\left|x_{n}\right| / 2\right\}} \frac{|w(x)-w(\bar{x})|^{2}}{|x-\bar{x}|^{n+2 s}} d x d \bar{x} \\
& +C \int_{Q_{1}} \int_{Q_{1} \cap\left\{\bar{x}:|x-\bar{x}|<\left|x_{n}\right| / 2\right\}}\left|x_{n}\right|^{1-2 s} \frac{|w(x)-w(\bar{x})|^{2}}{|x-\bar{x}|^{n+1}} d x d \bar{x}=: I_{1}+I_{2} \text {. }
\end{aligned}
$$

We bound $I_{1}$ using the $L^{\infty}$ estimate for $w$ and spherical coordinates centered at $x$ :

$$
\begin{gathered}
\int_{Q_{1}} \int_{Q_{1} \cap\left\{\bar{x}:|x-\bar{x}|>\left|x_{n}\right| / 2\right\}} \frac{|w(x)-w(\bar{x})|^{2}}{|x-\bar{x}|^{n+2 s}} d x d \bar{x} \leq C \int_{Q_{1}} d x \int_{\left|x_{n}\right| / 2}^{2 \sqrt{n}} d r \frac{1}{r^{2 s+1}} \\
\leq C \int_{-1}^{1} \frac{1}{\left|x_{n}\right|^{2 s}} d x_{n} \leq C
\end{gathered}
$$


Next, we consider $I_{2}$. By symmetry between $x_{n}$ and $-x_{n}$ we can suppose $x \in Q_{1}^{+}$. Using the gradient bounds (4.12) for $w$ and spherical coordinates centered at $x$, we get

$$
\begin{aligned}
I_{2} \leq & C \int_{Q_{1}^{+}} \int_{Q_{1} \cap\left\{\bar{x}:|x-\bar{x}|<x_{n} / 2\right\}} x_{n}^{1-2 s} \frac{|w(x)-w(\bar{x})|^{2}}{|x-\bar{x}|^{n+1}} d x d \bar{x} \\
& \leq C \int_{0}^{\varepsilon / 2} d x_{n} x_{n}^{1-2 s} \int_{0}^{x_{n} / 2} d r \frac{1}{\varepsilon^{2}}\left(\frac{\left|y_{n}(x, \bar{x})\right|}{\varepsilon}\right)^{2(2 s-1)} \\
& +C \int_{\varepsilon / 2}^{1} d x_{n} x_{n}^{1-2 s} \int_{0}^{x_{n} / 2} d r \frac{1}{y_{n}^{2}(x, \bar{x})},
\end{aligned}
$$

where $y(x, \bar{x}) \in B_{x_{n} / 2}(x)$ is a point of the segment joining $x$ and $\bar{x}$. In the first integral in the last bound, we have used that $y_{n}(x, \bar{x}) \leq x_{n}+x_{n} / 2 \leq 2 x_{n} \leq \varepsilon$. In the second integral we have used that, in case $y_{n}(x, \bar{x}) \leq \varepsilon, \frac{C}{\varepsilon}\left(\frac{y_{n}(x, \bar{x})}{\varepsilon}\right)^{2 s-1} \leq \frac{C}{y_{n}(x, \bar{x})}$ in (4.12).

Since $y(x, \bar{x}) \in B_{x_{n} / 2}(x)$, we have that $y_{n}(x, \bar{x}) \geq x_{n} / 2$ and thus we deduce that

$$
\begin{aligned}
& \int_{Q_{1}^{+}} \int_{\left\{\bar{x} \in Q_{1}:|x-\bar{x}|<x_{n} / 2\right\}} x_{n}^{1-2 s} \frac{|w(x)-w(\bar{x})|^{2}}{|x-\bar{x}|^{n+1}} d x d \bar{x} \\
& \quad \leq C \int_{0}^{\varepsilon / 2} d x_{n} x_{n}^{1-2 s} \int_{0}^{x_{n} / 2} d r \frac{1}{\varepsilon^{2}}\left(\frac{x_{n}}{\varepsilon}\right)^{2(2 s-1)}+C \int_{\varepsilon / 2}^{1} d x_{n} x_{n}^{1-2 s} \int_{0}^{x_{n} / 2} d r \frac{1}{x_{n}^{2}} \\
& \quad \leq C \frac{1}{\varepsilon^{4 s}} \int_{0}^{\varepsilon / 2} x_{n}^{2 s} d x_{n}+C \int_{\varepsilon / 2}^{1} x_{n}^{-2 s} d x_{n} \\
& \leq C \varepsilon^{1-2 s}+C \int_{\varepsilon / 2}^{1} x_{n}^{-2 s} d x_{n} \leq C \int_{\varepsilon}^{1} x_{n}^{-2 s} d x_{n} .
\end{aligned}
$$

Using (4.14), (4.15), and (4.16), we conclude that

$$
I \leq C \int_{\varepsilon}^{1} x_{n}^{-2 s} d x_{n}
$$

Case $1 / 2<s<1$. By (1.11) and (1.12) we must consider now the quantity

$$
\widetilde{I}:=\int_{Q_{1}^{-}} \int_{Q_{1}^{-}} \frac{|w(x)-w(\bar{x})|^{2}}{|x-\bar{x}|^{n+2 s}} d x d \bar{x}+\int_{Q_{1}^{+}} \int_{Q_{1}} x_{n}^{1-2 s} \frac{|w(x)-w(\bar{x})|^{2}}{|x-\bar{x}|^{n+1}} d x d \bar{x} .
$$

We recall that in this case, by (1.15) we have

$$
|D w(x)| \leq \begin{cases}\frac{c_{s}}{\varepsilon_{c}} & \text { in } A \cap\left\{\left|x_{n}\right| \leq \varepsilon\right\} \\ \frac{c_{s}}{\left|x_{n}\right|} & \text { in } A \cap\left\{\left|x_{n}\right|>\varepsilon\right\} \\ 28\end{cases}
$$


We have that

$$
\begin{aligned}
\widetilde{I} \leq & \int_{Q_{1}} \int_{Q_{1}} \frac{|w(x)-w(\bar{x})|^{2}}{|x-\bar{x}|^{n+2 s}} d x d \bar{x} \\
& +\int_{Q_{1}} \int_{Q_{1}}\left|x_{n}\right|^{1-2 s} \frac{|w(x)-w(\bar{x})|^{2}}{|x-\bar{x}|^{n+1}} d x d \bar{x} \\
\leq & C \int_{Q_{1}} \int_{Q_{1} \cap\left\{\bar{x}:|x-\bar{x}| \leq\left|x_{n}\right| / 2\right\}} \frac{|w(x)-w(\bar{x})|^{2}}{|x-\bar{x}|^{n+2 s}} d x d \bar{x} \\
& +C \int_{Q_{1}} \int_{Q_{1} \cap\left\{\bar{x}:|x-\bar{x}|>\left|x_{n}\right| / 2\right\}} \frac{|w(x)-w(\bar{x})|^{2}}{|x-\bar{x}|^{n+2 s}} d x d \bar{x} \\
\leq & C \int_{Q_{1}} \int_{Q_{1} \cap\left\{\bar{x}:|x-\bar{x}| \leq\left|x_{n}\right| / 2\right\}} \frac{|w(x)-w(\bar{x})|^{2}}{|x-\bar{x}|^{n+1}} d x d \bar{x} \\
& +C \int_{Q_{1}} \int_{Q_{1} \cap\left\{\bar{x}:\left|x_{n}\right| / 2<|x-\bar{x}| \leq \max \left\{\varepsilon / 2,\left|x_{n}\right| / 2\right\}\right\}}\left|x_{n}\right|^{1-2 s} \frac{|w(x)-w(\bar{x})|^{2}}{|x-\bar{x}|^{n+1}} d x d \bar{x} \\
& +C \int_{Q_{1}} \int_{Q_{1} \cap\left\{\bar{x}:|x-\bar{x}| \geq \max \left\{\varepsilon / 2,\left|x_{n}\right| / 2\right\}\right\}} \mid w\left(\left.x\right|^{1-2 s} \frac{|w(x)-w(\bar{x})|^{2}}{|x-\bar{x}|^{n+1}} d x d \bar{x}\right. \\
= & \widetilde{I}_{1}+\widetilde{I}_{2}+\widetilde{I}_{3} .
\end{aligned}
$$

We first bound $\widetilde{I}_{1}$. We have

$$
\widetilde{I}_{1} \leq C \int_{Q_{1}} \int_{Q_{1} \cap\left\{\bar{x}:|x-\bar{x}| \leq\left|x_{n}\right| / 2\right\}} \frac{|D w(y(x, \bar{x}))|^{2}}{|x-\bar{x}|^{n-2+2 s}} d x d \bar{x}
$$

where $y(x, \bar{x}) \in B_{\left|x_{n}\right| / 2}(x)$ is a point of the segment joining $x$ and $\bar{x}$. Now, the gradient bound (4.17) reads $|D w(y)| \leq c_{s} \min \left\{\varepsilon^{-1},\left|y_{n}\right|^{-1}\right\}$ for a.e. $y \in Q_{1}$. Since $y(x, \bar{x}) \in$ $B_{\left|x_{n}\right| / 2}(x)$, we have $\left|y_{n}(x, \bar{x})\right| \geq\left|x_{n}\right| / 2$ and $|D w(y(x, \bar{x}))| \leq c_{s} \min \left\{\varepsilon^{-1},\left|y_{n}(x, \bar{x})\right|^{-1}\right\} \leq$ $c_{s} \min \left\{\varepsilon^{-1}, 2\left|x_{n}\right|^{-1}\right\}$. Using spherical coordinates centered at $x$, we get

$$
\begin{aligned}
\widetilde{I}_{1} & \leq C \int_{Q_{1}} d x \int_{0}^{\left|x_{n}\right| / 2} d r r^{1-2 s} \min \left\{\frac{1}{\varepsilon^{2}}, \frac{1}{\left|x_{n}\right|^{2}}\right\} \\
& \leq C \int_{-1}^{1} d x_{n} \min \left\{\frac{1}{\varepsilon^{2}}, \frac{1}{\left|x_{n}\right|^{2}}\right\}\left|x_{n}\right|^{2-2 s} \\
& \leq C \int_{0}^{\varepsilon} \frac{1}{\varepsilon^{2}} x_{n}^{2-2 s} d x_{n}+C \int_{\varepsilon}^{1} x_{n}^{-2 s} d x_{n} \leq C \int_{\varepsilon}^{1} x_{n}^{-2 s} d x_{n} .
\end{aligned}
$$

Consider now $\widetilde{I}_{2}$. Here $\left|x_{n}\right|<\varepsilon$ (if not $\left\{\left|x_{n}\right| / 2<\max \left\{\left|x_{n}\right| / 2, \varepsilon / 2\right\}\right\}=\emptyset$ ). Using that $\widetilde{I}_{2}$ is symmetric in $x_{n}$ and $-x_{n}$, the gradient bound (4.17) and spherical coordinates centered at $x$ as for $\widetilde{I}_{1}$, we get

$$
\widetilde{I}_{2} \leq C \int_{0}^{\varepsilon} d x_{n} x_{n}^{1-2 s} \int_{x_{n} / 2}^{\varepsilon / 2} d r \frac{1}{\varepsilon^{2}} \leq C \varepsilon^{1-2 s} .
$$


Finally, using that $|w| \leq c_{s}$ in $Q_{1}$ and spherical coordinates centered at $x$, we get the following bound for $\widetilde{I}_{3}$ :

$$
\begin{aligned}
\widetilde{I}_{3} & \leq C \int_{Q_{1}} d x_{n}\left|x_{n}\right|^{1-2 s} \int_{\max \left\{\left|x_{n}\right| / 2, \varepsilon / 2\right\}}^{2 \sqrt{n}} d r \frac{1}{r^{2}} \\
& \leq C \int_{-1}^{1} d x_{n}\left|x_{n}\right|^{1-2 s} \min \left\{\frac{1}{\left|x_{n}\right|}, \frac{1}{\varepsilon}\right\} \\
& \leq C \int_{0}^{\varepsilon} \frac{1}{\varepsilon} x_{n}^{1-2 s} d x_{n}+C \int_{\varepsilon}^{1} x_{n}^{-2 s} d x_{n} \\
& \leq C \varepsilon^{1-2 s}+C \int_{\varepsilon}^{1} x_{n}^{-2 s} d x_{n} \leq C \int_{\varepsilon}^{1} x_{n}^{-2 s} d x_{n} .
\end{aligned}
$$

We conclude that

$$
\widetilde{I} \leq C \int_{\varepsilon}^{1} x_{n}^{-2 s} d x_{n}
$$

Step 2. Suppose now that $A$ is a Lipschitz subset of $\mathbb{R}^{n}$ or $A=\partial \Omega$, where $\Omega$ is an open bounded subset of $\mathbb{R}^{n+1}$ with Lipschitz boundary.

We consider a finite open covering $\left\{A_{r_{i} / 2}\right\}_{i=1, \ldots, m}=\left\{B_{r_{i} / 2} \cap A\right\}_{i=1, \ldots, m}$, where now $B_{r_{i} / 2}$ is the ball centered at $p_{i}$ (as in the proof of Theorem 1.7, case 2) but of radius $r_{i} / 2$. Here, for sake of simplicity, $B_{r_{i}}$ denotes both the ball in $\mathbb{R}^{n}$ or $\mathbb{R}^{n+1}$.

Let $\bar{\Gamma}$ be the closure of $\Gamma$ in $\mathbb{R}^{n}$ or $\mathbb{R}^{n+1}$. Only in the case $A \subset \mathbb{R}^{n}$, it may happen that $\bar{\Gamma} \backslash \Gamma \neq \emptyset$. In such case, for $p_{i} \in \bar{\Gamma} \backslash \Gamma$, there exists a radius $r_{i}$ and a bilipschitz diffeomorphism $\varphi_{i}: B_{r_{i}}\left(p_{i}\right) \rightarrow(-3,1) \times(-1,1)^{n-1}$ such that $\varphi_{i}\left(p_{i}\right)=(-1,0, \ldots, 0)$ and $\varphi_{i}$ satisfies properties (4.7), (4.8), and (4.9). We set $\varepsilon_{0}=\min \left\{r_{i} / 2,1 / 2\right\}$.

If $z$ and $\bar{z}$ are two points belonging to $A$ such that $|z-\bar{z}|<\varepsilon_{0}$, then there exists a set $A_{r_{i}}=B_{r_{i}} \cap A$ such that both $z$ and $\bar{z}$ belong to $A_{r_{i}}$. Hence

$$
\left\{(z, \bar{z}) \in A \times A:|z-\bar{z}|<\varepsilon_{0}\right\} \subset \bigcup_{i=1}^{m} A_{r_{i}} \times A_{r_{i}} .
$$

Let $L>1$ be a bound for the Lipschitz constants of all functions $\varphi_{1}, \ldots, \varphi_{m}, \varphi_{1}^{-1}, \ldots, \varphi_{m}^{-1}$. Let us first treat the case $0<\varepsilon \leq 1 /(2 L)$. 
We write

$$
\begin{aligned}
\iint_{B_{\mathrm{frac}}} & \frac{|w(z)-w(\bar{z})|^{2}}{|z-\bar{z}|^{n+2 s}} d \mathcal{H}^{n}(z) d \mathcal{H}^{n}(\bar{z}) \\
+ & \iint_{B_{\text {weig }}} d_{M}(z)^{1-2 s} \frac{|w(z)-w(\bar{z})|^{2}}{|z-\bar{z}|^{n+1}} d \mathcal{H}^{n}(z) d \mathcal{H}^{n}(\bar{z}) \\
= & \iint_{B_{\mathrm{frac}} \cap\left\{\bar{z}:|z-\bar{z}|<\varepsilon_{0}\right\}} \frac{|w(z)-w(\bar{z})|^{2}}{|z-\bar{z}|^{n+2 s}} d \mathcal{H}^{n}(z) d \mathcal{H}^{n}(\bar{z}) \\
& +\iint_{B_{\mathrm{frac}} \cap\left\{\bar{z}:|z-\bar{z}|>\varepsilon_{0}\right\}} \frac{|w(z)-w(\bar{z})|^{2}}{|z-\bar{z}|^{n+2 s}} d \mathcal{H}^{n}(z) d \mathcal{H}^{n}(\bar{z}) \\
& +\iint_{B_{\text {weig }} \cap\left\{\bar{z}:|z-\bar{z}|<\varepsilon_{0}\right\}} d_{M}(z)^{1-2 s} \frac{|w(z)-w(\bar{z})|^{2}}{|z-\bar{z}|^{n+1}} d \mathcal{H}^{n}(z) d \mathcal{H}^{n}(\bar{z}) \\
& +\iint_{B_{\text {weig }} \cap\left\{\bar{z}:|z-\bar{z}|>\varepsilon_{0}\right\}} d_{M}(z)^{1-2 s} \frac{|w(z)-w(\bar{z})|^{2}}{|z-\bar{z}|^{n+1}} d \mathcal{H}^{n}(z) d \mathcal{H}^{n}(\bar{z}) .
\end{aligned}
$$

Since $w$ is bounded and $\int_{B_{\text {weig }}} d_{M}(z)^{1-2 s} d z \leq C$ for every $0<s<1$, using spherical coordinates centered at $z$ as before, we have that

$$
\begin{aligned}
& \iint_{B_{\mathrm{frac}} \cap\left\{\bar{z}:|z-\bar{z}|>\varepsilon_{0}\right\}} \frac{|w(z)-w(\bar{z})|^{2}}{|z-\bar{z}|^{n+2 s}} d \mathcal{H}^{n}(z) d \mathcal{H}^{n}(\bar{z}) \\
& +\iint_{B_{\text {weig } \cap\left\{\bar{z}:|z-\bar{z}|>\varepsilon_{0}\right\}}} d_{M}(z)^{1-2 s} \frac{|w(z)-w(\bar{z})|^{2}}{|z-\bar{z}|^{n+1}} d \mathcal{H}^{n}(z) d \mathcal{H}^{n}(\bar{z}) \leq C .
\end{aligned}
$$

On the other hand, by the previous consideration,

$$
\begin{aligned}
& \iint_{B_{\text {frac }} \cap\left\{\bar{z}:|z-\bar{z}|<\varepsilon_{0}\right\}} \frac{|w(z)-w(\bar{z})|^{2}}{|z-\bar{z}|^{n+2 s}} d \mathcal{H}^{n}(z) d \mathcal{H}^{n}(\bar{z}) \\
& +\iint_{B_{\text {weig }} \cap\left\{\bar{z}:|z-\bar{z}|<\varepsilon_{0}\right\}} d_{M}(z)^{1-2 s} \frac{|w(z)-w(\bar{z})|^{2}}{|z-\bar{z}|^{n+1}} d \mathcal{H}^{n}(z) d \mathcal{H}^{n}(\bar{z}) \\
& \leq \sum_{i=1}^{m} \iint_{B_{\mathrm{frac}} \cap\left(A_{r_{i}} \times A_{r_{i}}\right)} \frac{|w(z)-w(\bar{z})|^{2}}{|z-\bar{z}|^{n+2 s}} d \mathcal{H}^{n}(z) d \mathcal{H}^{n}(\bar{z}) \\
& +\sum_{i=1}^{m} \iint_{B_{\text {weig }} \cap\left(A_{r_{i}} \times A_{r_{i}}\right)} d_{M}(z)^{1-2 s} \frac{|w(z)-w(\bar{z})|^{2}}{|z-\bar{z}|^{n+1}} d \mathcal{H}^{n}(z) d \mathcal{H}^{n}(\bar{z}) .
\end{aligned}
$$

If $A_{r_{i}} \cap \Gamma \neq \emptyset$ or $A_{r_{i}} \subset M$ then, by the construction of the open covering $\left\{A_{r_{i}}\right\}$, there exists a bilipschitz map $\varphi_{i}: B_{r_{i}} \rightarrow Q_{1} \times(-1,1)$ such that $\varphi_{i}\left(A_{r_{i}}\right)=Q_{1}$. Moreover, if $A_{r_{i}} \cap \Gamma \neq \emptyset$, we have also $\varphi_{i}\left(A_{r_{i}} \cap M\right)=\left\{x \in Q_{1}: x_{n}<0\right\}$. We use the bilipschitz map $\varphi_{i}$ to flatten the sets $B_{\text {frac }} \cap\left(A_{r_{i}} \times A_{r_{i}}\right)$ and $B_{\text {weig }} \cap\left(A_{r_{i}} \times A_{r_{i}}\right)$, and we set $v_{i}=w \circ \varphi_{i}^{-1}$. Given $x \in Q_{1}$, let $y=\varphi_{i}^{-1}(x) \in A_{r_{i}}$. Recalling that $L>1$ is a bound for the Lipschitz constants of all functions $\varphi_{1}, \ldots, \varphi_{m}, \varphi_{1}^{-1}, \ldots, \varphi_{m}^{-1}$, we have 
that $(1 / L) d_{\Gamma}(y) \leq\left|x_{n}\right| \leq L d_{\Gamma}(y)$ and $\left|D v_{i}(x)\right| \leq L|D w(y)|$. Therefore the gradient estimates (1.14) and (1.15) lead to the following bounds for $\left|D v_{i}\right|$ :

- for every $s \in(0,1 / 2]$,

$$
\begin{aligned}
\left|D v_{i}(x)\right| \leq L|D w(y)| & \leq \begin{cases}L \frac{c_{s}}{\varepsilon}\left(\frac{d_{\Gamma}(y)}{\varepsilon}\right)^{2 s-1} & \text { if } y \in A \text { and } d_{\Gamma}(y) \leq \varepsilon \\
L \frac{c_{s}}{d_{\Gamma}(y)} & \text { if } y \in A \text { and } d_{\Gamma}(y)>\varepsilon\end{cases} \\
& \leq \begin{cases}L \frac{c_{s}}{\varepsilon}\left(\frac{\left|x_{n}\right|}{L \varepsilon}\right)^{2 s-1} & \text { if } x \in Q_{1} \text { and }\left|x_{n}\right| \leq L \varepsilon \\
L^{2} \frac{c_{s}}{\left|x_{n}\right|} & \text { if } x \in Q_{1} \text { and }\left|x_{n}\right|>L \varepsilon\end{cases}
\end{aligned}
$$

- for every $s \in(1 / 2,1)$,

$$
\begin{aligned}
\left|D v_{i}(x)\right| \leq L|D w(y)| & \leq \begin{cases}L \frac{c_{s}}{\varepsilon} & \text { if } y \in A \text { and } d_{\Gamma}(y) \leq \varepsilon \\
L \frac{c_{s}}{d_{\Gamma}(y)} & \text { if } y \in A \text { and } d_{\Gamma}(y)>\varepsilon\end{cases} \\
& \leq \begin{cases}L \frac{c_{s}}{\varepsilon} & \text { if } x \in Q_{1} \text { and }\left|x_{n}\right| \leq L \varepsilon \\
L^{2} \frac{c_{s}}{\left|x_{n}\right|} & \text { if } x \in Q_{1} \text { and }\left|x_{n}\right|>L \varepsilon\end{cases}
\end{aligned}
$$

Thus we can apply the result proven in Step 1, with $\varepsilon$ replaced by $\varepsilon L$ (note that we have $\varepsilon L \leq 1 / 2$, as in Step 1$)$, to the function $v_{i} /\left(1+L^{2}\right)$. Using the Lipschitz property of $\varphi^{-1}$, we restate the conclusion for $w$ and we get

$$
\begin{aligned}
& \iint_{B_{\text {frac } \cap\left(A_{r_{i}} \times A_{r_{i}}\right)}} \frac{|w(z)-w(\bar{z})|^{2}}{|z-\bar{z}|^{n+2 s}} d \mathcal{H}^{n}(z) d \mathcal{H}^{n}(\bar{z}) \\
& +\iint_{B_{\text {weig }} \cap\left(A_{r_{i}} \times A_{r_{i}}\right)} d_{M}(z)^{1-2 s} \frac{|w(z)-w(\bar{z})|^{2}}{|z-\bar{z}|^{n+1}} d \mathcal{H}^{n}(z) d \mathcal{H}^{n}(\bar{z}) \leq C \int_{\varepsilon}^{1} \rho^{-2 s} d \rho .
\end{aligned}
$$

Last, we consider the case $A_{r_{i}} \cap M=\emptyset$. We recall that, in this case $r_{i}=\frac{1}{3} d_{M}\left(p_{i}\right)$, where $r_{i}$ and $p_{i}$ are respectively the radius and the center of the ball $B_{r_{i}}$. Then, for every $z \in A_{r_{i}}$, we have that $d_{\Gamma}(z) \geq d_{M}(z) \geq r_{i} \geq \varepsilon_{0}$ and thus we have that $|D w(z)| \leq \frac{c_{s}}{\varepsilon_{0}}$. 
Using this gradient bound to have $|D w(y(z, \bar{z}))| \leq C$, where $y(z, \bar{z})$ is a point of the segment joining $z$ and $\bar{z}$, and using also spherical coordinates, we get

$$
\begin{aligned}
\iint_{B_{\mathrm{frac}} \cap\left(A_{r_{i}} \times A_{r_{i}}\right)} \frac{|w(z)-w(\bar{z})|^{2}}{|z-\bar{z}|^{n+2 s}} d \mathcal{H}^{n}(z) d \mathcal{H}^{n}(\bar{z}) \\
\quad+\iint_{B_{\mathrm{weig}} \cap\left(A_{r_{i}} \times A_{r_{i}}\right)} d_{M}(z)^{1-2 s} \frac{|w(z)-w(\bar{z})|^{2}}{|z-\bar{z}|^{n+1}} d \mathcal{H}^{n}(z) d \mathcal{H}^{n}(\bar{z}) \\
\leq C \int_{A_{r_{i}}} d \mathcal{H}^{n}(z) \int_{0}^{r_{i}} d r \frac{1}{r^{2 s-1}}+C \int_{A_{r_{i}}} d \mathcal{H}^{n}(z) d_{M}(z)^{1-2 s} \int_{0}^{r_{i}} d r \leq C .
\end{aligned}
$$

Summing over $i=1, \ldots, m$, we conclude the proof in case $\varepsilon \leq 1 /(2 L)$.

Finally given $\varepsilon \in(0,1 / 2)$ with $\varepsilon>1 /(2 L)$, since (1.14) and (1.15) hold with such $\varepsilon$, they also hold with $\varepsilon$ replaced by $1 /(2 L)$. By the previous proof with $\varepsilon$ taken to be $1 /(2 L)$, the energy is bounded by

$$
C \int_{1 /(2 L)}^{1} \rho^{-2 s} d \rho \leq C \leq C \int_{\varepsilon}^{1} \rho^{-2 s} d \rho .
$$

\section{ENERGy ESTIMATE FOR GLOBAL MINIMIZERS}

In this section we give the proof of Theorem 1.2, which is based on a comparison argument. Let $v$ be a global minimizer of (1.2). The proof consists of 3 steps:

i) solving a Dirichlet problem, we construct an appropriate bounded comparison function $\bar{w}$ which takes the same values as $v$ on $\partial C_{R} \cap\{\lambda>0\}$ and thus, by minimality of $v$,

$$
\mathcal{E}_{s, C_{R}}(v) \leq \mathcal{E}_{s, C_{R}}(\bar{w})
$$

ii) we apply the extension Theorem 1.7 in the cylinder of radius $R$ and height $R$ to deduce

$$
\begin{array}{rl}
\int_{C_{R}} \lambda^{1-2 s}|\nabla \bar{w}|^{2} & d x d \lambda \leq C|| w \|_{L^{2}\left(\partial C_{R}\right)}^{2}+ \\
& +C \iint_{B_{\text {frac }}} \frac{|w(z)-w(\bar{z})|^{2}}{|z-\bar{z}|^{n+2 s}} d \mathcal{H}^{n}(z) d \mathcal{H}^{n}(\bar{z}) \\
& +C \iint_{B_{\text {weig }}} \lambda^{1-2 s} \frac{|w(z)-w(\bar{z})|^{2}}{|z-\bar{z}|^{n+1}} d \mathcal{H}^{n}(z) d \mathcal{H}^{n}(\bar{z}),
\end{array}
$$

where $z \in \partial C_{R}, w$ is the trace of $\bar{w}$ on $\partial C_{R}$ and $B_{\text {frac }}$ and $B_{\text {weig }}$ are defined as in (1.11) and (1.12), with $A=\partial C_{R}$ and $M=B_{R} \times\{0\}$.

iii) we prove, rescaling and using Theorem 1.9 , that the quantity in the right-hand side of (5.1) is bounded by $C R^{n-2 s} \int_{1 / R}^{1} \rho^{-2 s} d \rho$. 
Proof of Theorem 1.2. Let $v$ be a bounded global minimizer of (1.2). Let $u$ be its trace on $\partial \mathbb{R}_{+}^{n+1}$. Let $\tau \in[\inf u, \sup u]$ be such that $G(\tau)=c_{u}$, where $c_{u}$ as defined as in (1.5).

Throughout the proof, $C$ will denote positive constants depending only on $n, s$, $\|f\|_{C^{1, \gamma}}$ and $\|u\|_{L^{\infty}\left(\mathbb{R}^{n}\right)}$. As explained in (1.18), $v$ satisfies the following bounds:

$$
\begin{aligned}
& \left|\nabla_{x} v(x, \lambda)\right| \leq c_{s} \text { for every } x \in \mathbb{R}^{n} \text { and } \lambda \geq 0 \\
& |\nabla v(x, \lambda)| \leq \frac{c_{s}}{\lambda} \text { for every } x \in \mathbb{R}^{n} \text { and } \lambda>0 \\
& \left|\lambda^{1-2 s} \partial_{\lambda} v\right| \leq c_{s} \text { for every } x \in \mathbb{R}^{n} \text { and } \lambda>0 .
\end{aligned}
$$

We recall (see Remark 1.10) that the constants $c_{s}$ in (5.2) and (5.4) are not uniformly bounded for $s$ close to 0 and $s$ close to 1 respectively.

We estimate the energy $\mathcal{E}_{s, C_{R}}(v)$ of $v$ using a comparison argument. We define a function $\bar{w}=\bar{w}(x, \lambda)$ in $C_{R}$ in the following way. First we define $\bar{w}(x, 0)$ on the base of the cylinder to be equal to a smooth function $g(x)$ which is identically equal to $\tau$ in $B_{R-1}$ and $g(x)=v(x, 0)$ for $|x|=R$. The function $g$ is defined as follows:

$$
g=\tau \eta_{R}+\left(1-\eta_{R}\right) v(\cdot, 0)
$$

where $\eta_{R}$ is a smooth function depending only on $r=|x|$ such that $\eta_{R} \equiv 1$ in $B_{R-1}$ and $\eta_{R} \equiv 0$ outside $B_{R}$. Then we define $\bar{w}(x, \lambda)$ as the unique solution of the Dirichlet problem

$$
\begin{cases}\operatorname{div}\left(\lambda^{1-2 s} \nabla \bar{w}\right)=0 & \text { in } C_{R} \\ \bar{w}(x, 0)=g(x) & \text { on } B_{R} \times\{\lambda=0\} \\ \bar{w}(x, \lambda)=v(x, \lambda) & \text { on } \partial C_{R} \cap\{\lambda>0\} .\end{cases}
$$

Since $v$ is a global minimizer of $\mathcal{E}_{s, C_{R}}$ and $\bar{w}=v$ on $\partial C_{R} \times\{\lambda>0\}$, then

$$
\begin{aligned}
& d_{s} \int_{C_{R}} \frac{1}{2} \lambda^{1-2 s}|\nabla v|^{2} d x d \lambda+\int_{B_{R}}\left\{G(u(x, 0))-c_{u}\right\} d x \\
& \quad \leq d_{s} \int_{C_{R}} \frac{1}{2} \lambda^{1-2 s}|\nabla \bar{w}|^{2} d x d \lambda+\int_{B_{R}}\left\{G(\bar{w}(x, 0))-c_{u}\right\} d x .
\end{aligned}
$$

We prove now that

$$
d_{s} \int_{C_{R}} \frac{1}{2} \lambda^{1-2 s}|\nabla \bar{w}|^{2} d x d \lambda+\int_{B_{R}}\left\{G(\bar{w}(x, 0))-c_{u}\right\} d x \leq C R^{n-2 s} \int_{1 / R}^{1} \rho^{-2 s} d \rho .
$$

First of all, observe that the potential energy is bounded by $C R^{n-1}$. Indeed, by definition $\bar{w}(x, 0)=\tau$ in $B_{R-1}$, and therefore 


$$
\begin{gathered}
\int_{B_{R}}\left\{G(\bar{w}(x, 0))-c_{u}\right\} d x=\int_{B_{R} \backslash B_{R-1}}\left\{G(\bar{w}(x, 0))-c_{u}\right\} d x \\
\leq C\left|B_{R} \backslash B_{R-1}\right| \leq C R^{n-1} .
\end{gathered}
$$

Thus, we need to bound the Dirichlet energy. First of all, rescaling, we set

$$
\bar{w}_{1}(x, \lambda)=\bar{w}(R x, R \lambda)
$$

for $(x, \lambda) \in C_{1}=B_{1} \times(0,1)$. Moreover, if we set $\varepsilon=1 / R$ then

$$
\bar{w}_{1}(x, 0)= \begin{cases}\tau & \text { for }|x|<1-\varepsilon \\ v(R x, 0) & \text { for }|x|=1\end{cases}
$$

We observe that

$$
d_{s} \int_{C_{R}} \lambda^{1-2 s}|\nabla \bar{w}|^{2} d x d \lambda=d_{s} R^{n-2 s} \int_{C_{1}} \lambda^{1-2 s}\left|\nabla \bar{w}_{1}\right|^{2} d x d \lambda .
$$

Thus, it is enough to prove that

$$
d_{s} \int_{C_{1}} \lambda^{1-2 s}\left|\nabla \bar{w}_{1}\right|^{2} d x d \lambda \leq C \int_{\varepsilon}^{1} \rho^{-2 s} d \rho .
$$

Applying Theorem 1.7 (and Remark 1.8) with $\Omega=C_{1}$ and $M=B_{1} \times\{0\}$, we have that

$$
\begin{aligned}
& d_{s} \int_{C_{1}} \lambda^{1-2 s}\left|\nabla \bar{w}_{1}\right|^{2} d x d \lambda \\
& \quad \leq\left. C|| \bar{w}_{1}\right|_{L^{2}\left(\partial C_{1}\right)} ^{2}+C \iint_{B_{\text {frac }}} \frac{\left|\bar{w}_{1}(z)-\bar{w}_{1}(\bar{z})\right|^{2}}{|z-\bar{z}|^{n+2 s}} d \mathcal{H}^{n}(z) d \mathcal{H}^{n}(\bar{z}) \\
& \quad+C \iint_{B_{\text {weig }}} d_{M}(z)^{1-2 s} \frac{\left|\bar{w}_{1}(z)-\bar{w}_{1}(\bar{z})\right|^{2}}{\mid z-\bar{z}^{n+1}} d \mathcal{H}^{n}(z) d \mathcal{H}^{n}(\bar{z}),
\end{aligned}
$$

where $B_{\text {frac }}$ and $B_{\text {weig }}$ are defined as in (1.11) and (1.12) with $A=\partial C_{1}$ and $M=$ $B_{1} \times\{0\}$.

To bound the two double integrals above, we apply Theorem 1.9 to $\bar{w}_{\left.1\right|_{\partial C_{1}}}$ in $A=$ $\partial C_{1}$, taking $\Gamma=\partial B_{1} \times\{\lambda=0\}$. Since $\left|\bar{w}_{1}\right| \leq C$, we only need to check the gradient bounds (1.14) and (1.15) in $\partial C_{1}$. In the bottom boundary, $M=B_{1} \times\{0\}$, this is simple. Indeed $\bar{w}_{1} \equiv \tau$ in $B_{1-\varepsilon}$, and thus we need only to control $\left|\nabla \bar{w}_{1}(x, 0)\right|=$ $\varepsilon^{-1}|\nabla g(R x)| \leq C \varepsilon^{-1}$ for $|x|>1-\varepsilon$, where $g$ is defined in (5.5). We have used estimate (5.2) on $v$. Here $d_{\Gamma}(x)<\varepsilon$, and one can deduce that (1.14), (1.15) hold here. 
Next, to verify (1.14) and (1.15) in $\partial C_{1} \cap\{\lambda>0\}$ we use that $\bar{w}=v$ here and we know that $v$ satisfies (5.2), (5.3), and (5.4). Thus the tangential derivatives of $\bar{w}_{1}$ in $\partial C_{1} \cap\{\lambda>0\}$ satisfy

$$
\begin{gathered}
\left|\nabla_{x} \bar{w}_{1}(x, \lambda)\right| \leq c_{s} R=\frac{c_{s}}{\varepsilon} \text { for } \quad(x, \lambda) \in \partial C_{1} \cap\{\lambda>0\} \\
\left|\nabla \bar{w}_{1}(x, \lambda)\right| \leq \frac{c_{s} R}{R \lambda}=\frac{c_{s}}{\lambda} \text { for } \quad(x, \lambda) \in \partial C_{1} \cap\{\lambda>0\}
\end{gathered}
$$

and

$$
\left|\lambda^{1-2 s} \partial_{\lambda} \bar{w}_{1}(x, \lambda)\right| \leq \frac{c_{s} R}{R^{1-2 s}}=\frac{c_{s}}{\varepsilon^{2 s}} \text { for } \quad(x, \lambda) \in \partial C_{1} \cap\{\lambda>0\} .
$$

Estimate (5.10) is used on the top boundary $B_{1} \times\{\lambda=1\}$ to verify in this set (1.14) and (1.15). Note that here $d_{\Gamma}((x, \lambda))$ is comparable to $\lambda=1$ up to multiplicative constants. (5.9), (5.10), and (5.11) also lead to (1.14) and (1.15) on the lateral boundary $\partial B_{1} \times(0,1)$, where $d_{\Gamma}((x, \lambda))=\lambda$. Hence, $\bar{w}_{\left.1\right|_{\partial C_{1}}}$ satisfies the hypothesis of Theorem 1.9. We conclude that the estimate for the Dirichlet energy (5.8) holds.

\section{Energy estimate For monotone Solutions in $\mathbb{R}^{3}$}

In section 5 of [4], we proved two technical lemmas which led to the energy estimate for monotone solutions (without limit assumption) and $s=1 / 2$ in dimension $n=$ 3. Here we give analogue results but for every fractional power $0<s<1$ of the Laplacian.

The first lemma concerns the stability property of the limit functions

$$
\underline{v}\left(x_{1}, x_{2}, \lambda\right):=\lim _{x_{3} \rightarrow-\infty} v(x, \lambda) \text { and } \bar{v}\left(x_{1}, x_{2}, \lambda\right):=\lim _{x_{3} \rightarrow+\infty} v(x, \lambda),
$$

and some properties of the potential $G$ in relation with these functions. The second proposition establishes that monotone solutions are global minimizers among a suitable class of functions which in turn allows us to apply a comparison argument to obtain energy estimates.

Lemma 6.1. Let $f$ be a $C^{1, \gamma}$ function, for some $\gamma>\max \{0,1-2 s\}$, and $u$ be $a$ bounded solution of equation (1.1) in $\mathbb{R}^{3}$, such that $u_{x_{3}}>0$. Let $v$ be the s-extension of $u$ in $\mathbb{R}_{+}^{4}$.

Set

$$
\underline{v}\left(x_{1}, x_{2}, \lambda\right):=\lim _{x_{3} \rightarrow-\infty} v(x, \lambda) \text { and } \bar{v}\left(x_{1}, x_{2}, \lambda\right):=\lim _{x_{3} \rightarrow+\infty} v(x, \lambda) .
$$

Then, $\underline{v}$ and $\bar{v}$ are solutions of $(1.2)$ in $\mathbb{R}_{+}^{3}$, and each of them is either constant or it depends only on $\lambda$ and on one Euclidian variable in the $\left(x_{1}, x_{2}\right)$-plane. As a consequence, each $\underline{u}=\underline{v}(\cdot, 0)$ and $\bar{u}=\bar{v}(\cdot, 0)$ is either constant or $1-D$.

Moreover, set $m=\inf \underline{u} \leq \widetilde{m}=\sup \underline{u}$ and $\widetilde{M}=\inf \bar{u} \leq M=\sup \bar{u}$. 
Then, $G>G(\widetilde{m})=G(m)$ in $(m, \widetilde{m}), G^{\prime}(\widetilde{m})=G^{\prime}(m)=0$ and $G>G(\widetilde{M})=G(M)$ in $(\widetilde{M}, M), G^{\prime}(\widetilde{M})=G^{\prime}(M)=0$.

Proof. The proof is the same as in the case of the half-Laplacian (see [4]). We do not supply all details and just recall the two main steps:

(1) show that the functions $\underline{v}$ and $\bar{v}$ are stable solutions of problem (1.2) in $\mathbb{R}_{+}^{3}$ and thus their trace in $\mathbb{R}^{2}$ is 1 -D by the one-dimensional symmetry result of the first author and Sire, Theorem 2.12 of [6];

(2) apply Theorem 2.2 (i) of the first author and Sire [5], which gives necessary conditions on the nonlinearities $f$ for which there exists an increasing solution to (1.2) in dimension $n=1$. This leads to the conditions on $G$ stated at the end of the lemma.

Proposition 6.2. Let $f$ be any $C^{1, \gamma}$ nonlinearity, for some $\gamma>\max \{0,1-2 s\}$. Let $u$ be a bounded solution of (1.1) in $\mathbb{R}^{n}$ such that $u_{x_{n}}>0$, and let $v$ be the s-extension of $u$ in $\mathbb{R}_{+}^{n+1}$.

Then,

$$
\begin{aligned}
d_{s} \int_{C_{R}} \frac{1}{2} \lambda^{a}|\nabla v(x, \lambda)|^{2} d x d \lambda+ & \int_{B_{R}} G(v(x, 0)) d x \\
& \leq d_{s} \int_{C_{R}} \frac{1}{2} \lambda^{a}|\nabla w(x, \lambda)|^{2} d x d \lambda+\int_{B_{R}} G(w(x, 0)) d x
\end{aligned}
$$

for every $H^{1}\left(C_{R}, \lambda^{a}\right)$ function $w$ such that $w=v$ on $\partial^{+} C_{R}=\partial C_{R} \cap\{\lambda>0\}$ and $\underline{v} \leq w \leq \bar{v}$ in $C_{R}$, where $\underline{v}$ and $\bar{v}$ are defined by

$$
\underline{v}\left(x^{\prime}, \lambda\right):=\lim _{x_{n} \rightarrow-\infty} v\left(x^{\prime}, x_{n}, \lambda\right) \text { and } \bar{v}\left(x^{\prime}, \lambda\right):=\lim _{x_{n} \rightarrow+\infty} v\left(x^{\prime}, x_{n}, \lambda\right) .
$$

Proof. As in the case of the half-Laplacian, this property of local minimality of monotone solutions $w$ such that $\underline{v} \leq w \leq \bar{v}$ follows from the following two results:

i) Uniqueness of the solution to the problem

$$
\begin{cases}\operatorname{div}\left(\lambda^{a} \nabla w\right)=0 & \text { in } C_{R}, \\ w=v & \text { on } \partial^{+} C_{R}=\partial C_{R} \cap\{\lambda>0\}, \\ -d_{s} \lambda^{a} \partial_{\lambda} w=f(w) & \text { on } B_{R}, \\ \underline{v} \leq w \leq \bar{v} & \text { in } C_{R} .\end{cases}
$$

Thus, the solution must be $w \equiv v$. This is the analogue of Lemma 3.1 of [7] for $s=1 / 2$, and below we comment on its proof. In this fractional case, it is stated in Lemma 5.1 of [6]. 
ii) Existence of an absolute minimizer for (6.1), that is, for $\mathcal{E}_{s, C_{R}}$ in the set

$$
C_{v}=\left\{w \in H^{1}\left(C_{R}, \lambda^{a}\right) \mid w \equiv v \text { on } \partial^{+} C_{R}, \underline{v} \leq w \leq \bar{v} \text { in } C_{R}\right\} .
$$

The statement of the proposition follows from the fact that by i) and ii), the monotone solution $v$, by uniqueness, must agree with the absolute minimizer in $C_{R}$.

To prove points i) and ii), we proceed exactly as in [6]. For this, it is important that $\underline{v}$ and $\bar{v}$ are respectively, a strict subsolution and a strict supersolution of the Dirichlet-Neumann mixed problem (6.1). We make a short comment about these proofs.

i) The proof of uniqueness is based on sliding the function $v(x, \lambda)$ in the direction $x_{n}$. We set

$$
v^{t}\left(x_{1}, \ldots, x_{n}, \lambda\right)=v\left(x_{1}, \ldots, x_{n}+t, \lambda\right) \quad \text { for every }(x, \lambda) \in \bar{C}_{R} .
$$

Since $v^{t} \rightarrow \bar{v}$ as $t \rightarrow+\infty$ uniformly in $\bar{C}_{R}$ and $\underline{v}<w<\bar{v}$ (here we use that $w$ solves (6.1) and that $\underline{v}$ is a subsolution to guarantee $\underline{v}<w$ ), then $w<v^{t}$ in $\bar{C}_{R}$, for $t$ large enough. We want to prove that $w<v^{t}$ in $\bar{C}_{R}$ for every $t>0$. Suppose that $s>0$ is the infimum of those $t>0$ such that $w<v^{t}$ in $\bar{C}_{R}$. Then by applying maximum principle and Hopf's lemma we get a contradiction, since one would have $w \leq v^{s}$ in $\bar{C}_{R}$ and $w=v^{s}$ at some point in $\bar{C}_{R} \backslash \partial^{+} C_{R}$.

ii) To prove the existence of an absolute minimizer for $\mathcal{E}_{C_{R}}$ in the convex set $C_{v}$, we proceed exactly as in Lemma 4.1 of [6], substituting -1 and +1 by the subsolutions and supersolution $\underline{v}$ and $\bar{v}$, respectively.

We give now the proof of the energy estimate in dimension 3 for monotone solutions without the limit assumptions.

Proof of Theorem 1.4. We follow the proof of Theorem 5.2 of [1]. We need to prove that the comparison function $\bar{w}$, used in the proof of Theorem $\underline{1.2}$, satisfies $\underline{v} \leq \bar{w} \leq$ $\bar{v}$. Then we can apply Proposition 6.2 to make the comparison argument with the function $\bar{w}$ (as for global minimizers). We recall that $\bar{w}$ is the solution of

$$
\begin{cases}\operatorname{div}\left(\lambda^{1-2 s} \nabla \bar{w}\right)=0 & \text { in } C_{R} \\ \bar{w}(x, 0)=g(x) & \text { on } B_{R} \times\{\lambda=0\} \\ \bar{w}(x, \lambda)=v(x, \lambda) & \text { on } \partial C_{R} \cap\{\lambda>0\},\end{cases}
$$

where $g=\tau \eta_{R}+\left(1-\eta_{R}\right) v(\cdot, 0)$ as in (5.5). Recall that $\tau$ is such that $G(\tau)=c_{u}=$ $\min \left\{G(s): \inf _{\mathbb{R}^{n}} u \leq s \leq \sup _{\mathbb{R}^{n}} u\right\}$. Thus, if we prove that we van take $\tau$ such that 
$\sup \underline{v} \leq \tau \leq \inf \bar{v}$, then $\underline{v} \leq g \leq \bar{v}$ and hence $\underline{v}$ and $\bar{v}$ are respectively, subsolution and supersolutions of (6.2). It follows that $\underline{v} \leq \bar{w} \leq \bar{v}$, as desired.

To show that $\sup \underline{v} \leq \tau \leq \inf \bar{v}$, let $m=\inf u=\inf \underline{u}$ and $M=\sup u=\sup \bar{u}$, where $\underline{u}$ and $\bar{u}$ are defined in Lemma 6.1. Set $\widetilde{m}=\sup \underline{u}$ and $\widetilde{M}=\inf \bar{u}$; obviously $\widetilde{m}$ and $\widetilde{M}$ belong to $[m, M]$. By Lemma $6.1, \underline{u}$ and $\bar{u}$ are either constant or monotone 1-D solutions, moreover

$$
G>G(m)=G(\widetilde{m}) \quad \text { in }(m, \widetilde{m})
$$

in case $m<\tilde{m}$ (i.e. $\underline{u}$ not constant), and

$$
G>G(M)=G(\widetilde{M}) \text { in }(\widetilde{M}, M)
$$

in case $\widetilde{M}<M$ (i.e. $\bar{u}$ not constant).

In all four possible cases (that is, each $\underline{u}$ and $\bar{u}$ is constant or one-dimensional), we deduce from (6.3) and (6.4) that $\widetilde{m} \leq \widetilde{M}$ and that there exists $\tau \in[\widetilde{m}, \widetilde{M}]=[\sup \underline{v}=$ $\sup \underline{u}, \inf \bar{v}=\inf \bar{u}]$ such that $G(\tau)=c_{u}$ (recall that $c_{u}$ is the infimum of $G$ in the range of $u$ ), as desired.

\section{1-D SYMMETRY IN $\mathbb{R}^{3}$}

To prove Theorem 1.5 we follow the argument, used by Ambrosio and the first author [3] in their proof of the conjecture of De Giorgi in dimension $n=3$. It relies on a Liouville type theorem. We recall an adapted version of this result for the fractional case, given by the first author and Sire (Theorem 4.10 in [5]).

Theorem 7.1. (5]) Let $a \in(-1,1), \varphi \in L_{\text {loc }}^{\infty}\left(\overline{\mathbb{R}_{+}^{n+1}}\right)$ be a positive function and suppose that $\sigma \in H_{\text {loc }}^{1}\left(\overline{\mathbb{R}_{+}^{n+1}}, \lambda^{a}\right)$ is a solution of

$$
\begin{cases}-\sigma \operatorname{div}\left(\lambda^{a} \varphi^{2} \nabla \sigma\right) \leq 0 & \text { in } \mathbb{R}_{+}^{n+1} \\ -\sigma \lambda^{a} \frac{\partial \sigma}{\partial \lambda} \leq 0 & \text { on } \partial \mathbb{R}_{+}^{n+1}\end{cases}
$$

in the weak sense. Moreover assume that for every $R>1$,

$$
\int_{C_{R}} \lambda^{a}(\varphi \sigma)^{2} d x d \lambda \leq C R^{2}
$$

for some constant $C$ independent of $R$.

Then, $\sigma$ is constant.

We can now give the proof of our one-dimensional symmetry result.

Proof of Theorem 1.5. We follow the proof of Theorem 1.4 in [4], where the result was established for $s=1 / 2$. Hence, here we may assume $s>1 / 2$. 
First of all observe that both global minimizers and monotone solutions are stable. Thus, in both cases (see Lemma 6.1 of [6]), there exists a Hölder continuous function $\varphi$ in $\overline{\mathbb{R}_{+}^{4}}$ such that $\varphi>0$ in $\overline{\mathbb{R}_{+}^{4}}, \varphi \in H_{\text {loc }}^{1}\left(\overline{\mathbb{R}_{+}^{4}}, \lambda^{a}\right)$, and

$$
\begin{cases}\operatorname{div}\left(\lambda^{a} \nabla \varphi\right)=0 & \text { in } \mathbb{R}_{+}^{4} \\ -d_{s} \lambda^{a} \partial_{\lambda} \varphi=f^{\prime}(v) \varphi & \text { on } \partial \mathbb{R}_{+}^{4} .\end{cases}
$$

Note that, if $u$ is a monotone solution in the direction $x_{3}$, say, then we can choose $\varphi=v_{x_{3}}$, where $v$ is the $s$-extension of $u$ in the half space. For $i=1,2,3$ fixed, consider the function:

$$
\sigma_{i}=\frac{v_{x_{i}}}{\varphi} .
$$

We prove that $\sigma_{i}$ is constant in $\mathbb{R}_{+}^{4}$ using the Liouville type Theorem 7.1 and our energy estimate.

We have that

$$
\operatorname{div}\left(\lambda^{a} \varphi^{2} \nabla \sigma_{i}\right)=0 \quad \text { in } \mathbb{R}_{+}^{4} .
$$

Moreover $-\lambda^{a} \partial_{\lambda} \sigma_{i}$ is zero on $\partial \mathbb{R}_{+}^{4}$. Indeed

$$
\lambda^{a} \varphi^{2} \partial_{\lambda} \sigma_{i}=\lambda^{a} \varphi v_{\lambda x_{i}}-\lambda^{a} v_{x_{i}} \varphi_{\lambda}=0
$$

since both $v_{x_{i}}$ and $\varphi$ satisfy the same boundary condition

$$
-d_{s} \lambda^{a} \partial_{\lambda} v_{x_{i}}-f^{\prime}(v) v_{x_{1}}=0, \quad-d_{s} \lambda^{a} \partial_{\lambda} \varphi-f^{\prime}(v) \varphi=0 .
$$

By Theorems 1.2 and 1.4, $v$ satisfies the energy estimate (1.9). Since $G(u)-c_{u} \geq 0$ in $\mathbb{R}^{3}$, we deduce

$$
\int_{C_{R}} \lambda^{1-2 s}\left(\varphi \sigma_{i}\right)^{2} \leq \int_{C_{R}} \lambda^{1-2 s}|\nabla v|^{2} \leq C R^{2}, \quad \text { for every } R>2 \text { and } 1 / 2<s<1 .
$$

Thus, using Theorem 7.1, we deduce that $\sigma_{i}$ is constant for every $i=1,2,3$. We get

$$
v_{x_{i}}=c_{i} \varphi \text { for some constant } c_{i} \text {, with } i=1,2,3 .
$$

We conclude the proof observing that if $c_{1}=c_{2}=c_{3}=0$ then $v$ is constant. Otherwise we have

$$
c_{i} v_{x_{j}}-c_{j} v_{x_{i}}=0 \text { for every } i \neq j,
$$

and we deduce that $v$ depends only on $\lambda$ and on the variable parallel to the vector $\left(c_{1}, c_{2}, c_{3}\right)$. Thus $u(x)=v(x, 0)$ is 1-D. 


\section{REFERENCES}

[1] G. Alberti, L. Ambrosio, and X. Cabré, On a long-standing conjecture of E. De Giorgi: symmetry in 3D for general nonlinearities and a local minimality property, Acta Appl. Math. 65 (2001), 9-33.

[2] G. Alberti, G. Bouchitté, and S. Seppecher, Phase transition with the line-tension effect, Arch. Rational Mech. Anal. 144 (1998), 1-46.

[3] L. Ambrosio and X. Cabré, Entire solutions of semilinear elliptic equations in $\mathbb{R}^{3}$ and a conjecture of De Giorgi, Journal Amer. Math. Soc. 13 (2000), 725-739.

[4] X. Cabré and E. Cinti, Energy estimates and 1-D symmetry for nonlinear equations involving the half-Laplacian, Discrete Contin. Dyn. Syst. 28 (2010), 1179-1206.

[5] X. Cabré and Y. Sire, Nonlinear equations for fractional Laplacians I: regularity, maximum principles, and Hamiltonian estimates, to appear in Analysis \& PDE. Preprint, arXiv: 1012.0867.

[6] X. Cabré and Y. Sire, Nonlinear equations for fractional Laplacians II: existence, uniqueness, and qualitative properties of solutions, to appear in Trans. Amer. Math. Soc. Preprint, arXiv: 1111.0796 .

[7] X. Cabré and J. Solà-Morales, Layer solutions in a halph-space for boundary reactions, Comm. Pure and Appl. Math. 58 (2005), 1678-1732.

[8] L. Caffarelli, J.-M. Roquejoffre, and O. Savin, Nonlocal minimal surfaces, Comm. Pure Appl. Math. 63 (2010), 1111-1144.

[9] L. Caffarelli and L. Silvestre, An extension problem related to the fractional Laplacian, Comm. Part. Diff. Eq. 32 (2007), 1245-1260.

[10] L. Caffarelli and P.E. Souganidis, Convergence of nonlocal threshold dynamics approximations to front propagation, Arch. Ration. Mech. Anal. 195 (2010), 1-23.

[11] L. Caffarelli and E. Valdinoci, Regularity properties of nonlocal minimal surfaces via limiting arguments, preprint, arXiv: 1105.1158.

[12] E. Cinti, Bistable Elliptic Equations With Fractional Diffusion, Ph.D. Thesis. Universitat Politècnica de Catalunya and Università di Bologna, 2010.

[13] M. del Pino, M. Kowalczyk, and J. Wei, On De Giorgi Conjecture in dimension $N \geq 9$, Ann. of Math. 174 (2011), 1485-1569.

[14] E. B. Fabes, C. E. Kenig, and R. P. Serapioni, The local regularity of solutions of degenerate elliptic equations, Comm. Partial Differential Equations 7 (1982), 77-116.

[15] R. L. Frank and E. Lenzmann, Uniqueness and nondegeneracy of ground states for $(-\Delta)^{s} Q+$ $Q-Q^{\alpha+1}=0$ in $\mathbb{R}$, preprint, arXiv: 1009.4042.

[16] N. Ghoussoub and C. Gui, On a conjecture of De Giorgi and some related problems, Math. Ann. 311 (1998), 481-491.

[17] M.d.M. González, Gamma convergence of an energy functional related to the fractional Laplacian, Calc. Var. Part. Diff. Eq. 36 (2009), 173-210.

[18] J.L. Lions and E. Magenes, Non-Homogeneous Boundary Value Problems and Applications I, Springer-Verlag (1972).

[19] L. Moschini, New Liouville theorems for linear second order degenerate elliptic equations in divergence form, Ann. I. H. Poincaré 22 (2005), 11-23. 
[20] A. Nekvinda, Characterization of traces of the weighted Sobolev space $H_{\varepsilon, M}^{1, p}$, Funct. Approx. Comment. Math 20 (1992), 143-151.

[21] A. Nekvinda, Characterization of traces of the weighted Sobolev space $W^{1, p}\left(\Omega, d_{M}^{\varepsilon}\right)$ on $M$, Czechoslovak Mth. J. 43 (1993), 713-722.

[22] G. Palatucci, O. Savin, and E. Valdinoci, Local and global minimizers for a variational energy involving a fractional norm, Annali di Matematica Pura ed Applicata (2012), Doi: 10.1007/s10231-011-0243-9.

[23] O. Savin, Phase ransitions: regularity of flat level sets, Ann. of Math. 169 (2009), 41-78.

[24] O. Savin and E. Valdinoci, Density estimates for a variational model driven by the Gagliardo norm, preprint, http://arxiv.org/abs/1007.2114

[25] O. Savin and E. Valdinoci, Г-convergence for nonlocal phase transitions, preprint, http://arxiv.org/abs/1007.1725

[26] O. Savin and E. Valdinoci, Regularity of nonlocal minimal cones in dimension 2, preprint, http://www.ma.utexas.edu/mp arc-bin/mpa?yn=12-8

[27] L. Silvestre, Regularity of the obstacle problem for a fractional power of the Laplace operator, Comm. Pure Appl. Math. 60, 1, 67-112.

[28] Y. Sire and E. Valdinoci, Fractional Laplacian phase transitions and boundary reactions: A geometric inequality and a symmetry result, Jour. Functional Analysis 256 (2009), 1842-1864.

[29] P.R. Stinga and J. L. Torrea, Extension problem and Harnack's inequality for some fractional operators, Comm. Partial Differential Equations 35 (2010), 2092-2122.

ICREA and Departament de Matemàtica Aplicada 1, Universitat Politècnica de Catalunya, Diagonal 647, 08028 Barcelona (Spain)

E-mail address: xavier.cabre@upc.edu

Dipartimento di Matematica "F. Casorati", Università degli Studi di Pavia, Via Ferrata 1, 27100 PaVia (Italy)

E-mail address: eleonora.cinti@unipv.it 